\title{
Theoretical study of the line profiles of the hydrogen perturbed by collisions with protons
}

\author{
M. G. Santos ${ }^{\star}$ and S. O. Kepler \\ Instituto de Física, Universidade Federal do Rio Grande do Sul, 91501-900 Porto Alegre, RS, Brazil
}

Accepted 2012 January 24. Received 2012 January 17; in original form 2011 September 22

\begin{abstract}
We present theoretical calculations of the quasi-molecular line profiles for the Lyman (Ly $\alpha$, $\mathrm{Ly} \beta, \mathrm{Ly} \gamma$ and $\mathrm{Ly} \delta)$ and Balmer $(\mathrm{H} \alpha, \mathrm{H} \beta, \mathrm{H} \gamma, \mathrm{H} \delta, \mathrm{H} \epsilon, \mathrm{H} 8, \mathrm{H} 9$ and $\mathrm{H} 10)$ series perturbed by collisions with protons. In all calculations, we include the dependence of the dipole moments as a function of the internuclear distance during the collision. The broadening from ion collisions must be added to the normal electron Stark broadening.
\end{abstract}

Key words: line: profiles - white dwarfs.

\section{INTRODUCTION}

The satellite lines present in the far-wing profiles of the Lyman and Balmer series lines of atomic hydrogen have been observed in stars and laboratory plasmas (Kielkopf, Allard \& Decrette 2002). Experimentally, the primary difficulties in studying these line profiles are (1) the development of techniques to observe neutral atomic hydrogen interactions at densities high enough for spectral line broadening effects to be significant and (2) quantitatively characterizing the conditions under which the spectra are created. On the theoretical side, the difficulty arises in developing methods for computing the contributions from all possible molecular states of $\mathrm{H}_{2}$ and $\mathrm{H}_{2}^{+}$to the spectrum of free atoms in collision.

Satellite features at 1600 and $1405 \AA$ in the $\mathrm{Ly} \alpha$ wing associated with free-free quasi-molecular transitions of $\mathrm{H}_{2}$ and $\mathrm{H}_{2}^{+}$are observed in the ultraviolet (UV) spectra of a few stars obtained with the International Ultraviolet Explorer (IUE) and the Hubble Space Telescope (HST). The satellites in the red wing of $\operatorname{Ly} \beta$ are in the 905-1187 ̊ spectral region, covered by Far Ultraviolet Spectroscopic Explorer (FUSE). The stars which show Lyman satellites are DA white dwarfs (WDs), old horizontal branch stars of spectral type A and $\lambda$ Bootis stars (Allard et al. 2000). WDs are the end product of evolution of $\sim 98$ per cent of all stars (e.g. Kepler et al. 2007). Having consumed all the available nuclear fuel (which depends on the individual stellar mass), their source of luminosity is dominated by only the residual internal thermal energy. This radiates away, exponentially cooling the star, but due to the small surface area (a typical WD radius is $\sim 10^{9} \mathrm{~cm}$ ), cooling times are long, taking approximately $10^{10}$ years for their effective temperatures to decrease from around $10^{5}$ to near $10^{3} \mathrm{~K}$. Therefore, WDs are old, and hidden in their structure is a historical record of stellar formation and evolution in our Galaxy.

UV spectroscopic observations of WDs reveal a line shape very different from the expected simple Stark broadening, with satellite lines in the red wing of $\operatorname{Ly} \beta$ near 1078 and $1060 \AA$ (Koester et al.

^E-mail: marcios@if.ufrgs.br
1996, 1998). The strengths of these satellite features, and indeed the entire shape of the wings in the Lyman series, are very sensitive to the degree of ionization in the stellar atmosphere or laboratory plasma, because it is the ionization that determines the relative importance of broadening by ion and neutral collisions. Therefore, the satellites may be used as a temperature diagnostic in a plasma where the Saha ionization equilibrium holds. In the case of Ly $\alpha$ and the $\mathrm{H}-\mathrm{H}^{+} \mathrm{Ly} \beta$ satellites, the shape of the potential plays a dominant role in the large difference in the broadening of quasimolecular features (Allard et al. 1998b).

Quasi-molecular transitions occur when a photon is absorbed or emitted by a hydrogen atom while this atom interacts with one or more neighbouring particles, which may be atoms, ions or molecules (Allard \& Kielkopf 1982). At short distances, the interaction between the perturbed atoms and the other particle(s) usually leads to the formation of satellite molecular lines on the wings of atomic lines (Allard, Kielkopf \& Feautrier 1998a; Allard et al. 1998b, 1999). Structures observed in the wing of $\mathrm{Ly} \alpha$ at 1623 and $1405 \AA$ are due to absorptions of quasi-molecular $\mathrm{H}_{2}$ and $\mathrm{H}_{2}^{+}$, respectively. The intensity of these two satellites depends strongly on the degree of ionization in the stellar atmosphere and consequently on the effective temperature $T_{\text {eff }}$ and surface gravity $\log g$ (Holweger, Koester \& Allard 1994).

The atmosphere of a WD comprises less than $10^{-13}$ of the stellar mass and is dominated by hydrogen (DA WDs) or helium (DB/DO) and sometimes shows small amounts of heavy elements (e.g. DAZ, DBZ and DOZ WDs) (see e.g. Koester 2010). The primary technique to determine $T_{\text {eff }}$ and $\log g$ of WDs is by comparing observed spectra with synthetic spectra from theoretical atmosphere models (e.g. Tremblay \& Bergeron 2009). The advantage of this spectroscopic technique is that the theoretical line profiles are extremely sensitive to variations in the atmospheric parameters. Bergeron, Saffer \& Liebert (1992) were one of the first to apply this to a large number of WDs; they fitted the hydrogen Balmer lines. The technique was later applied to the UV Lyman lines (e.g. Kepler \& Nelan 1993; Bergeron et al. 1995; Vennes et al. 2005). 
Bergeron \& Leggett (2002) made improvements to the models by taking into account the optical quasi-molecular opacities due to the dipole moments induced by the interaction between particles. This interaction causes a temporary rearrangement of electronic charges, which forms a temporary molecule, thus producing absorptions or emissions beyond those of the isolated atoms.

Currently there are a large number of optical spectra of WDs that have been taken as part of the Sloan Digital Sky Survey (SDSS; e.g. Eisenstein et al. 2006; Kleinman 2010), allowing for the study of the WD mass distribution (Kepler et al. 2007). Kleinman et al. (2004) and Liebert, Bergeron \& Holberg (2005) derive masses from spectroscopic fits, and Bergeron, Wesemael \& Fontaine (1991) and Koester (1991) discuss the apparent increase in the average mass of DAs with $T_{\text {eff }}<12000 \mathrm{~K}$. Kepler et al. (2006) compare SDSS spectra with high signal-to-noise ratio $(\mathrm{S} / \mathrm{N})$ spectra obtained with the 8-m Gemini telescope for four WDs with $T_{\text {eff }} \sim 12000 \mathrm{~K}$ and determine that the masses derived from fitting the relatively low $\mathrm{S} / \mathrm{N}$ SDSS spectra are systematically overestimated by $\Delta M \simeq 0.13 \mathrm{M}_{\odot}$. Due to a correlation between $T_{\text {eff }}$ and $\log g$ (a small increase in $T_{\text {eff }}$ can be offset by a small decrease in $\log g$ ), such discrepancies are concentrated only in the region around the maximum of the Balmer lines, $14000 \geq T_{\text {eff }} \geq 12000 \mathrm{~K}$. To study the trend of the apparent increased average mass of DAs, Kepler et al. (2010) analyse 1505 such WDs with $\mathrm{S} / \mathrm{N} \geq 20$ and $T_{\text {eff }}>12000 \mathrm{~K}$ and determined an average DA mass of $M / \mathrm{M}_{\odot}=0.604 \pm 0.003$. They observe that the distribution of the sample is similar to that of the Palomar Green survey published by Liebert et al. (2005). Falcon et al. (2010) used an ensemble average of gravitational redshifts of 449 DAs, which is independent of line profiles, and estimated a mean mass of $0.640 \pm 0.014 \mathrm{M}_{\odot}$.

Koester et al. (2009), Koester (2010), Tremblay \& Bergeron (2009) and Gianninas et al. (2010) study the possible causes for this apparent increase in mass, not seen in photometric determinations (Engelbrecht \& Koester 2007; Kepler et al. 2007). They conclude this phenomenon is not caused by He contamination, but Tremblay et al. (2011) propose that the use of one-dimensional calculations of convection in the atmosphere, compared to more realistic threedimensional calculations, might be the cause. There is a huge effort to eliminate the inconsistencies in the model calculations, and these attempts show the need for continued study of the interaction of hydrogen perturbed by protons, electrons, atoms and molecules at high pressure.

The goal of our work is to study the interaction of the hydrogen atom with protons, which temporarily creates the $\mathrm{H}_{2}^{+}$molecule. We calculate the potential of $\mathrm{H}_{2}^{+}$up to $n=10$, and analyse all possible transitions that can occur, as well as the dipole moments for all possible transitions. In the literature, we find calculations only for $\operatorname{Ly} \alpha$, $\operatorname{Ly} \beta$ and $\operatorname{Ly} \gamma$ and $\mathrm{H} \alpha$; we calculate these at increased resolution. These new theoretical data must be inserted in the modelling of line profiles at high density, in addition to the electron Stark broadening.

\section{THEORETICAL ANALYSIS}

The dipole moments induced by the interaction between radiator and perturber cause a temporary repositioning of electronic charges, which temporarily forms a molecule. This leads to the possible appearance of satellite features in the wing of the line profile and produces absorptions or emissions different from those of noninteracting atoms (Frommhold 1993).

The position of the satellite line depends directly on the extreme of the potential difference of the transition $\Delta V_{\text {ext }}(R)$ and the dependence of the potential difference $\Delta V(R)=V_{j}(R)-V_{i}(R)$ with internuclear separation $R$ of the states $i$ and $j$ of the allowed transitions of $\mathrm{H}_{2}^{+}$; the intensity and shape of the satellites depend on both the potential difference and the radiative dipole moment (Allard et al. 2004).

To calculate spectral line profiles affected by collisions with protons, we must know precisely the theoretical potential that describes the molecular interaction between radiator and perturber, as well as the changes in dipole momenta due to the radioactive atom-ion separation for each molecular state.

Madsen \& Peek (1971) calculate the values of the potential of $\mathrm{H}_{2}^{+}$only for states with $n \leq 3$. Allard et al. (2004) calculate the potential for states with $n \leq 4$, which allows them to calculate the line profile for $\mathrm{Ly} \gamma$.

The values of the dipole moments of $\mathrm{H}_{2}^{+}$that are available in the literature were calculated by Ramaker \& Peek (1972, 1973), and Allard, Noselidze \& Kruk (2009) refers to the calculation of $n=4$.

In this paper, we calculate all potential and dipole moments up to $n \leq 10$ of $\mathrm{H}_{2}^{+}$as a function of the internuclear distance $R$ up to 300 au (atomic units), with a step of $0.01 \mathrm{au}$. We compare the potential of the $n \leq 3$ states with those in the literature (Madsen \& Peek 1971) and show differences smaller than $10^{-13}$, the precision quoted in the published values. We also compare the computed dipole moments with those tabulated by Ramaker \& Peek (1973) and those plotted in Allard et al. (2009); they are fully consistent.

\subsection{Potentials for the hydrogen molecule $\mathrm{H}_{2}^{+}$}

The structure of the molecular ion $\mathrm{H}_{2}^{+}$has been studied by Teller (1930), Hylleraas (1931), Jaffé (1934), Baber \& Hassé (1935), Bates $\&$ Poots (1953) and Madsen \& Peek (1971), but accurate tables of the electronic energy as a function of the internuclear distance for the excited states $n \geq 3$ have not been published.

As originally shown by Burrau (1927), the Schrödinger equation for $\mathrm{H}_{2}^{+}$can be separated and the electronic energy $E$ calculated exactly. The Schrödinger equation of the electron in atomic units is

$\frac{1}{2} \nabla^{2} \Psi+\left(E+\frac{1}{r_{a}}+\frac{1}{r_{b}}-\frac{1}{R}\right) \Psi=0$,

where $r_{a}$ and $r_{b}$ are the distances measured in units of $a_{0}$ of the electron to the two positive charges $a$ and $b, E$ is the energy measured in units of Hartree $E_{\mathrm{h}}$, and $R$ is the internuclear separation.

In terms of confocal elliptical coordinates, defined by $\lambda=\left(r_{a}+\right.$ $\left.r_{b}\right) / R, \mu=\left(r_{a}-r_{b}\right) / R$ and $\phi$, the azimuthal angle with respect to the internuclear axis, the Schrödinger equation can then be separated in three equations:

$\frac{\partial^{2} \Phi}{\partial \phi^{2}}+m^{2} \Phi=0$

$\frac{\partial}{\partial \mu}\left[\left(1-\mu^{2}\right) \frac{\partial M}{\partial \mu}\right]+\left(-A+p^{2} \mu^{2}-\frac{m^{2}}{1-\mu^{2}}\right) M=0$

and

$\frac{\partial}{\partial \lambda}\left[\left(\lambda^{2}-1\right) \frac{\partial \Lambda}{\partial \lambda}\right]+\left(A+2 R \lambda-p^{2} \lambda^{2}-\frac{m^{2}}{\lambda^{2}-1}\right) \Lambda=0$,

where $\Psi(\lambda, \mu, \phi)=\Lambda(\lambda) M(\mu) \Phi(\phi), p^{2}=-\varepsilon R^{2} / 2, \varepsilon=E-(1 / R)$ is the electronic energy, and $A$ and $m$ are the constants of separation.

The solution of equation (2) is direct, i.e.

$\Phi=\mathrm{e}^{( \pm i m \phi)}$. 
The solution of equation (3), the angular component, is obtained using the Hylleraas (1931) method:

$M(\mu)=\sum_{s} c_{s} P_{m+s}^{m}(\mu)$,

where $P_{m+s}^{m}(\mu)$ are the generalized Legendre polynomials of first order. The summation extends only over even or odd values of $s$, depending on the state calculated.

Applying equation (6) in equation (3), we obtain a recurrence relation for the coefficients $c_{s}$,

$a_{s} c_{s-2}+b_{s} c_{s}+d_{s} c_{s+2}=0$,

where

$$
\begin{aligned}
a_{s}= & p^{2} \frac{(s-1) s}{(2 s+2 m-3)(2 s+2 m-1)}, \\
b_{s}= & -A-(s+m)(s+m+1) \\
& +p^{2}\left[\frac{(s+1)(s+1+2 m)}{(2 s+2 m+3)(2 s+2 m+1)}\right. \\
& \left.+\frac{s(s+2 m)}{(2 s+2 m+1)(2 s+2 m-1)}\right], \\
d_{s}= & p^{2} \frac{(s+2 m+2)(s+2 m+1)}{(2 s+2 m+5)(2 s+2 m+3)} .
\end{aligned}
$$

The recurrence relation (7) forms an infinite set of homogeneous linear equations whose solution requires the determinant of the coefficient matrix to be null.

The solution of equation (4) for the radial part is obtained using the method of Jaffé (1934):

$\Lambda(\lambda)=\left(\lambda^{2}-1\right)^{m / 2}(1+\lambda)^{\sigma} \mathrm{e}^{-p \lambda} \sum_{t=0}^{\infty} g_{t}\left(\frac{\lambda-1}{\lambda+1}\right)^{t}$,

where $\sigma=(R / p)-m-1$.

Applying equation (8) to equation (4), the following recurrence equation is found:

$\alpha_{t} g_{t+1}-\beta_{t} g_{t}+\gamma_{t} g_{t-1}=0$,

where

$$
\begin{aligned}
& \alpha_{t}=(t+1)(t+m+1), \\
& \beta_{t}=2 t^{2}+(4 p-2 \sigma) t-A+p^{2}-2 p \sigma-(m+1)(m+\sigma), \\
& \gamma_{t}=(t-1-\sigma)(t-1-\sigma-m) .
\end{aligned}
$$

The recurrence relation (9) also produces an infinite set of homogeneous linear equations, whose determinant of the matrix of coefficients must also be null. When calculating the determinant of the infinite recurrence relations of equations (7) and (9), we obtain two functions $f_{1}(A, \varepsilon, R)$ and $f_{2}(A, \varepsilon, R)$, which depend on $A, \varepsilon$ and $R$. The electronic energy (potential energy $\left.V_{i}\right) \varepsilon$ is obtained by calculating the solution of both functions $f_{1}(A, \varepsilon, R)$ and $f_{2}(A, \varepsilon$, $R)$, e.g. by using the Newton-Raphson method. Practical considerations require the truncation of the recurrence relations (9) and (7). We choose $t=s=25$ for the data presented here, because larger values for $t$ and $s$ do not produce any significant change in $A$ and $\varepsilon$. The coefficients $c_{s}$ and $g_{t}$ are calculated using the method of continued fractions.

\subsection{Electric dipole moments}

Choosing the origin of the coordinate system in the centre of the line segment which unites the two positive charges and taking this line as the axis $z$, the matrix element to be evaluated of the components of the electric dipole moment, $D_{i j}$, is specified by

$D_{i j}=e\langle i|\boldsymbol{r}| j\rangle=e Q_{i, j}(\boldsymbol{r})$,

where $e$ represents the electron charge, $\boldsymbol{r}$ is the position vector of the electron relative to a molecular fixed frame, in coordinates $x, y$ and $z$ of the electron, $\Psi_{i}$ and $\Psi_{j}$ are the electronic functions in the Born-Oppenheimer approximation of the states $i$ and $j$, and $Q_{i, j}(\boldsymbol{r})$ are the matrix elements.

The matrix elements $Q_{i, j}(\boldsymbol{r})$ of the various components of the electric dipole moment according to Ramaker \& Peek (1973) are specified by

$Q_{i, j}(\boldsymbol{r})=\int \Psi_{i}^{*}(\lambda, \mu, \phi)(\boldsymbol{r}) \Psi_{j}(\lambda, \mu, \phi) \mathrm{d} \tau$,

where $\tau$ is the volume element. $Q_{i, j}(\boldsymbol{r})$ is obtained by inserting equations (5), (6) and (8) into equation (11). The matrix elements $Q_{i, j}(\boldsymbol{r})$ can be written as a function of the coordinates $x, y$ and $z$ as follows:

$$
\begin{aligned}
Q_{i, j}(x)= & N_{i} N_{j}\left(\frac{R}{2}\right)^{4} \int_{0}^{2 \pi} \Phi_{i}^{*}(\phi) \cos (\phi) \Phi_{j}(\phi) \mathrm{d} \phi \\
& \times\left[\Omega_{i, j}\left(\frac{1}{2}, 0, \frac{1}{2}, 2\right)-\Omega_{i, j}\left(\frac{1}{2}, 2, \frac{1}{2}, 0\right)\right], \\
Q_{i, j}(y)= & N_{i} N_{j}\left(\frac{R}{2}\right)^{4} \int_{0}^{2 \pi} \Phi_{i}^{*}(\phi) \sin (\phi) \Phi_{j}(\phi) \mathrm{d} \phi \\
& \times\left[\Omega_{i, j}\left(\frac{1}{2}, 0, \frac{1}{2}, 2\right)-\Omega_{i, j}\left(\frac{1}{2}, 2, \frac{1}{2}, 0\right)\right], \\
Q_{i, j}(z)= & N_{i} N_{j}\left(\frac{R}{2}\right)^{4} \int_{0}^{2 \pi} \Phi_{i}^{*}(\phi) \Phi_{j}(\phi) \mathrm{d} \phi \\
& \times\left[\Omega_{i, j}(0,1,0,3)-\Omega_{i, j}(0,3,0,1)\right],
\end{aligned}
$$

where $\Omega_{i, j}(a, b, c, d)$ is defined by

$$
\begin{aligned}
\Omega_{i, j}(a, b, c, d)= & \int_{-1}^{1} M_{i}^{*}(\mu)\left(1-\mu^{2}\right)^{a} \mu^{b} M_{j}(\mu) \mathrm{d} \mu \\
& \times \int_{1}^{\infty} \Lambda_{i}^{*}(\lambda)\left(\lambda^{2}-1\right)^{c} \lambda^{d} \Lambda_{j}(\lambda) \mathrm{d} \lambda .
\end{aligned}
$$

The normalization constants can be calculated from

$$
\begin{aligned}
N_{i}^{-2}= & \left(\frac{R}{2}\right)^{3}\left[\Omega_{i, i}(0,0,0,2)-\Omega_{i, i}(0,2,0,0)\right] \\
& \times \int \Phi_{i}^{*}(\phi) \Phi_{i}(\phi) \mathrm{d} \phi,
\end{aligned}
$$

$$
\begin{aligned}
N_{j}^{-2}= & \left(\frac{R}{2}\right)^{3}\left[\Omega_{j, j}(0,0,0,2)-\Omega_{j, j}(0,2,0,0)\right] \\
& \times \int \Phi_{j}^{*}(\phi) \Phi_{j}(\phi) \mathrm{d} \phi .
\end{aligned}
$$

After calculating the values of $Q_{i, j}(\boldsymbol{r})$ for each components $x, y$ and $z$, we obtain $D_{i j}$ as follows (Herman \& Wallis 1956):

$\left|D_{i j}\right|^{2}=\left|e Q_{i, j}(x)\right|^{2}+\left|e Q_{i, j}(y)\right|^{2}+\left|e Q_{i, j}(z)\right|^{2}$.

We compute the values of the electric dipole moments, equation (18), for all transitions of the Lyman and Balmer series for the $\mathrm{H}_{2}^{+}$molecular ion for internuclear separations up to 300 au. 
Table 1. Comparison between the data of Ramaker \& Peek (1973) (column 3) and this work (column 4) of the electric dipole moments for Ly $\alpha$ of $\mathrm{H}_{2}^{+}$in atomic units.

\begin{tabular}{lccc}
\hline$R\left(a_{0}\right)$ & Transition & $Q_{\alpha, \beta}(R)$ & $Q_{\alpha, \beta}(R)$ \\
\hline 1.0 & $2 p \pi_{\mathrm{u}}-1 s \sigma_{\mathrm{g}}$ & 0.5494 & 0.5493774 \\
11.0 & $2 p \pi_{\mathrm{u}}-1 s \sigma_{\mathrm{g}}$ & 0.7169 & 0.7168555 \\
60.0 & $2 p \pi_{\mathrm{u}}-1 s \sigma_{\mathrm{g}}$ & 0.7450 & 0.7449931 \\
1.0 & $3 p \sigma_{\mathrm{u}}-1 s \sigma_{\mathrm{g}}$ & 0.1651 & 0.1651107 \\
11.0 & $3 p \sigma_{\mathrm{u}}-1 s \sigma_{\mathrm{g}}$ & 0.4859 & 0.4858565 \\
80.0 & $3 p \sigma_{\mathrm{u}}-1 s \sigma_{\mathrm{g}}$ & 0.5207 & 0.5207119 \\
1.0 & $4 f \sigma_{\mathrm{u}}-1 s \sigma_{\mathrm{g}}$ & 0.001055 & 0.0010541 \\
11.0 & $4 f \sigma_{\mathrm{u}}-1 s \sigma_{\mathrm{g}}$ & 0.6609 & 0.6608712 \\
80.0 & $4 f \sigma_{\mathrm{u}}-1 s \sigma_{\mathrm{g}}$ & 0.5326 & 0.5325508 \\
1.0 & $2 s \sigma_{\mathrm{g}}-2 p \sigma_{\mathrm{u}}$ & 1.241 & 1.2406089 \\
11.0 & $2 s \sigma_{\mathrm{g}}-2 p \sigma_{\mathrm{u}}$ & 0.4844 & 0.4844248 \\
80.0 & $2 s \sigma_{\mathrm{g}}-2 p \sigma_{\mathrm{u}}$ & 0.5207 & 0.5207119 \\
1.0 & $3 d \sigma_{\mathrm{g}}-2 p \sigma_{\mathrm{u}}$ & 1.010 & 1.0104411 \\
11.0 & $3 d \sigma_{\mathrm{g}}-2 p \sigma_{\mathrm{u}}$ & 0.6349 & 0.6349347 \\
80.0 & $3 d \sigma_{\mathrm{g}}-2 p \sigma_{\mathrm{u}}$ & 0.5326 & 0.5325508 \\
1.0 & $3 d \pi_{\mathrm{g}}-2 p \sigma_{\mathrm{u}}$ & 0.8584 & 0.8584398 \\
11.0 & $3 d \pi_{\mathrm{g}}-2 p \sigma_{\mathrm{u}}$ & 0.7935 & 0.7935359 \\
60.0 & $3 d \pi_{\mathrm{g}}-2 p \sigma_{\mathrm{u}}$ & 0.7450 & 0.7449931 \\
\hline
\end{tabular}

Table 1 shows values of the electric dipole moments of this work compared with those of Ramaker \& Peek (1973) for some transitions, demonstrating that our work presents a higher precision and greater coverage of the internuclear distance.

For equations (6) and (8) to be solutions of the appropriate electronic functions $\Psi_{i}$, the summations must be allowed to go to infinity. However, we achieve convergence of the series for $s=31$ and $t=10$.

The data of the potential and electric dipole moments are plotted in subsequent sections for the Lyman series ( $\operatorname{Ly} \alpha$ to $\operatorname{Ly} \delta$ ) and Balmer $\alpha$. Electric dipole moments are plotted for Balmer $\alpha$ and Balmer $\beta$.

\section{PROFILES AND SATELLITES}

Currently there are different approaches to the calculation of the line profiles, namely quasi-static, quantum mechanical and unified theory.

In the quasi-static approximation, the expression of the crosssection may be written as (Margenau \& Lewis 1959; Allard \& Kielkopf 1982; Rohrmann, Althaus \& Kepler 2011)

$\sigma_{i j}(\nu)=\frac{16}{3} \pi^{3} n_{\mathrm{p}} \alpha\left\{\frac{h \nu}{\left|\mathrm{d} V_{i j} / \mathrm{d} R\right|}\right\} D_{i j} R^{2} \mathrm{e}^{\left(4 \pi n_{\mathrm{p}} R^{3} / 3\right)} \mathrm{e}^{-\beta E_{i}(R)}$,

where $\beta=1 / k T, k$ is the Boltzmann constant, and $T$ is the gas temperature. $n_{\mathrm{p}}$ is the mean density of perturbers in the gas, $V_{i j}$ is the potential energy difference associated with a transition with an energy of $h v=V_{i j} \equiv V_{j}(R)-V_{i}(R)$ at the internuclear separation $R, D_{i j}(R)$ is the dipole moment, and $E_{i}(R)$ corresponds to the change in energy (with respect to $R=\infty$ ) in the state $i$ of $\mathrm{H}_{2}^{+}$. When there is an extreme in the curve corresponding to the difference in the potential energies between the upper and lower states, $\mathrm{d} V_{i j} / \mathrm{d} R$ is zero. Therefore, if the derivative is zero, there is a divergence called a satellite line.

In the quantum mechanical approach, the cross-section for the spontaneous plus stimulated association process is given by

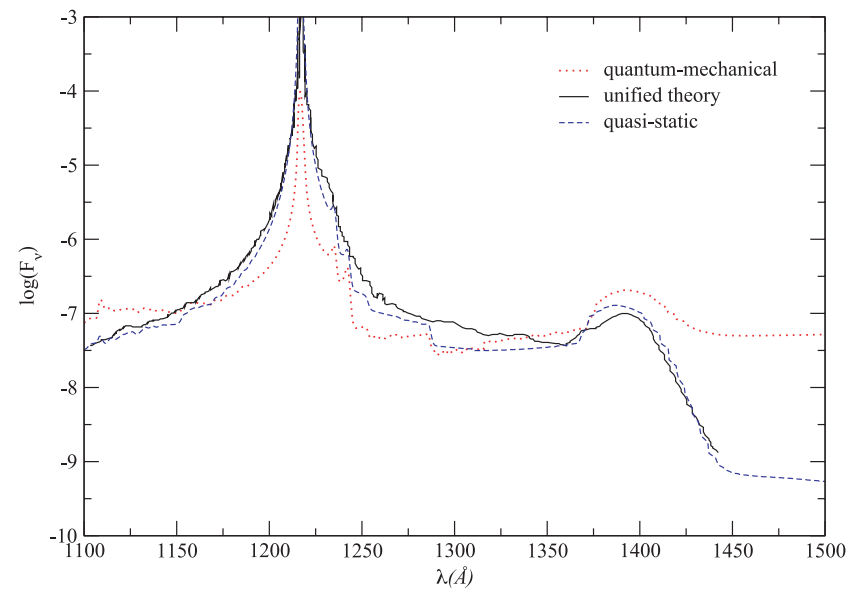

Figure 1. Comparison of the line profile of Ly $\alpha$ normalized between the three approaches, quasi-static (dashed line), quantum mechanical (dotted line) and unified theory (solid line) adapted from Allard et al. (1999).

(Zygelman \& Dalgarno 1990)

$$
\begin{aligned}
\sigma(E)= & \sum_{N^{\prime}} \sum_{v^{\prime \prime}} \frac{64}{3} \frac{\pi^{5}}{c^{3}} \frac{v^{3}}{\kappa^{2}} \frac{1}{1-\exp (-h v / k T)} \\
& \times p\left[N^{\prime} M_{v^{\prime \prime}, N^{\prime}-1 ; \kappa, N^{\prime}}^{2}+\left(N^{\prime}+1\right) M_{v^{\prime \prime}, N^{\prime}+1 ; \kappa, N^{\prime}}^{2}\right],
\end{aligned}
$$

where $E$ is the relative collision energy, $\kappa$ is the wavenumber of relative motion, $p$ is the probability of approach in the initial electronic state, $N^{\prime}$ is the initial rotational quantum number, $v^{\prime \prime}$ is the final vibrational quantum number, and $M$ is the electric dipole matrix element connecting the initial continuum and final rotationalvibrational states of the ground electronic state.

The unified theory has been developed in Allard et al. (1999), and a detailed discussion is presented there. The fundamental expression of the normalized spectrum line $F_{v}(\Delta v)$ is computed from the Fourier transform (Allard et al. 2009)

$F_{v}(\Delta v)=\frac{1}{\pi} \operatorname{Re} \int_{0}^{+\infty} \Phi(s) \mathrm{e}^{-\mathrm{i} \Delta v s} \mathrm{~d} s$.

Fig. 1 shows the comparison of the line profile of Ly $\alpha$ of the three approaches mentioned above. The solid line shows the approach from the unified theory, which has a resolution determined by Fourier transform (equation 21), while the quantum mechanical approaches (dashed line) and quasi-static (solid line) values provide similar but distinct profiles due to the calculation of the derivative by the numerical difference in the quasi-static and by the series expansion in quantum mechanics. The three profiles are similar; however, the quasi-static approach better represents the correct global shape of the line wings and position of the satellites, in comparison with previously published data (Allard et al. 1999). As the main aims of our work are the calculations of the electric dipole moments, the potentials of $\mathrm{H}_{2}^{+}$ion, the calculations of the global shape of line profiles and the location of the satellites, we use the quasi-static approach to calculate the line profiles. Unfortunately, the quasi-static approach does not provide an appropriate shape of the centre of the lines; thus, there is a need for better modelling of the centre lines of each transition.

Our interest is to find the correct position of the satellites in the wings of the line profile and their general form (i.e. the divergences in the profile) and bring these results to the attention of astrophysicists. If they are observable, they will be specific for protons (although other ions may give qualitatively similar effects). 
To obtain the profiles, we used the method of Rohrmann et al. (2011). For lines profile of the Lyman and Balmer series in a quasistatic approach, we identified each pair of levels $(i, j)$ of the quasimolecule $\mathrm{H}-\mathrm{H}^{+}$that contributes to the formation of a given line. Only transitions between states $\mathrm{u}$ (ungerade) and g (gerade) are allowed, i.e. with opposite parity and taking $\left|m-m^{\prime}\right|=0$ or 1 as the allowed dipole. The second step is to calculate the effective cross-section $\sigma_{i j}(v)$, equation (19), using the potentials and dipole moments for the transitions already identified. For the calculation of $\sigma_{i j}(\nu)$, we need to analyse the frequencies $v$ that arise from the difference in energy between these levels $h v=V_{j}-V_{i}$. However, we must take into account that different internuclear distances can produce the same frequency. The effective cross-section is the sum of each of these contributions.

The line profile is the sum of the effective cross-sections $\sigma_{i j}(v)$ multiplied by the statistical weights of each of the respective transitions. The calculated profile was convolved with a Maxwellian distribution of velocities to take into account the thermal Doppler broadening.

The line profiles and satellite lines of Lyman and Balmer series shown in this paper are for single densities of $10^{16}$ to $10^{18} \mathrm{~cm}^{-3}$, typical of the atmosphere of WD stars and that can be produced in z-pinch laboratories. Recall that the real profile must include the electrons Stark broadening, which do not have satellites.

\subsection{Lyman series}

\subsubsection{Lyman $\alpha$}

The Ly $\alpha$ total profile depends on six individual transitions. The line profile calculations shown in Figs 2 and 3 have been carried out for temperatures of 4000 and $12000 \mathrm{~K}$, at proton densities of $10^{16}, 10^{17}$ and $10^{18} \mathrm{~cm}^{-3}$.

Fig. 4 shows the potential energy for the transitions that contribute to the profile of Ly $\alpha$ and Fig. 5 shows the electronic transition dipole moments for Ly $\alpha$ due to $\mathrm{H}$ perturbed by collisions with protons.

Table 2 lists the wavelengths of the satellite lines with the upper and lower state identifications and the distance of the atom-ion pair at which the potential extreme occurs. Several of these features can be identified in the total Ly $\alpha$ profile of Figs 2 and 3.

We list in Table 2 the satellite around $\lambda=1400 \AA$, observed by Koester et al. (1985) and Nelan \& Wegner (1985), who proposed that the lines are caused by the quasi-molecular absorption of the $\mathrm{H}_{2}^{+}$molecule.

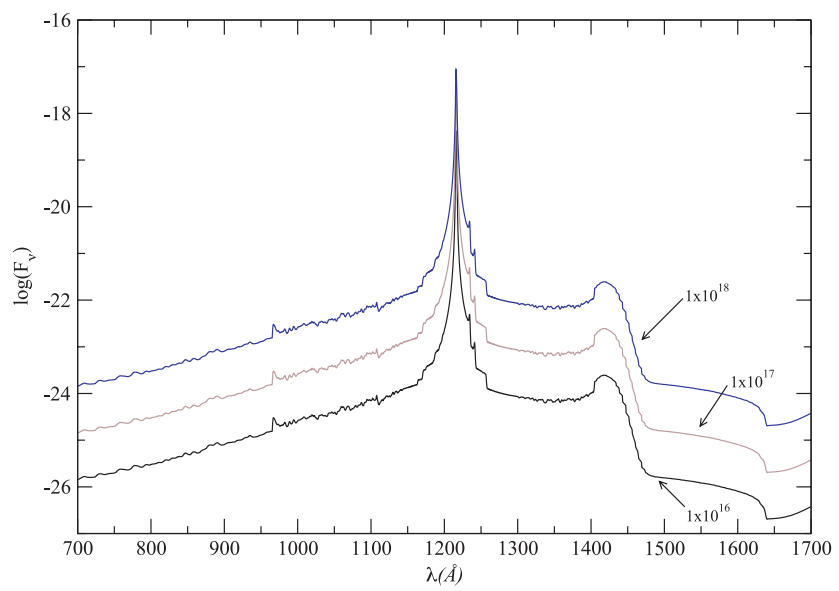

Figure 2. Ly $\alpha$ profile with density of $\mathrm{H}^{+}$perturbers $(T=4000 \mathrm{~K}), n_{\mathrm{H}}^{+}=$ $10^{16}, 10^{17}$ and $10^{18} \mathrm{~cm}^{-3}$ (from bottom to top).

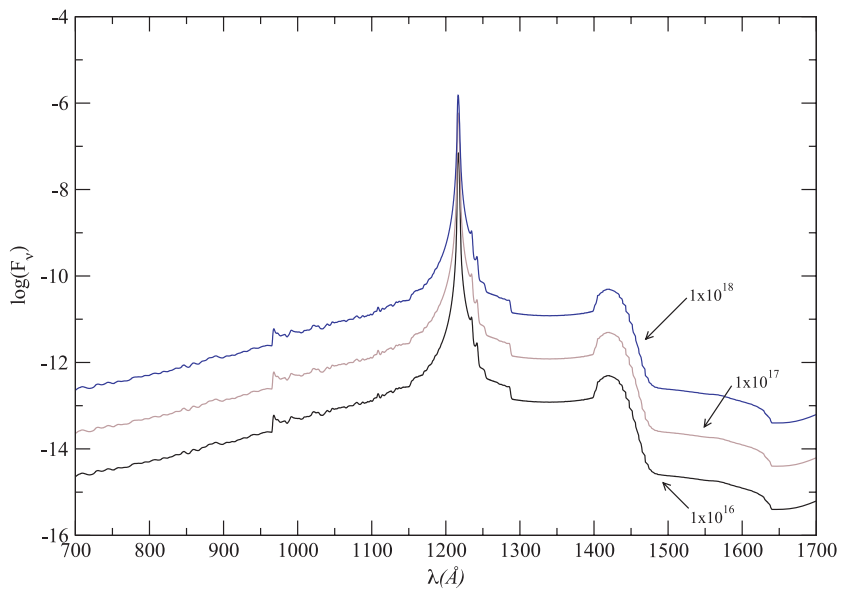

Figure 3. Ly $\alpha$ profile with density of $\mathrm{H}^{+}$perturbers $(T=12000 \mathrm{~K}), n_{\mathrm{H}}^{+}=$ $10^{16}, 10^{17}$ and $10^{18} \mathrm{~cm}^{-3}$ (from bottom to top).

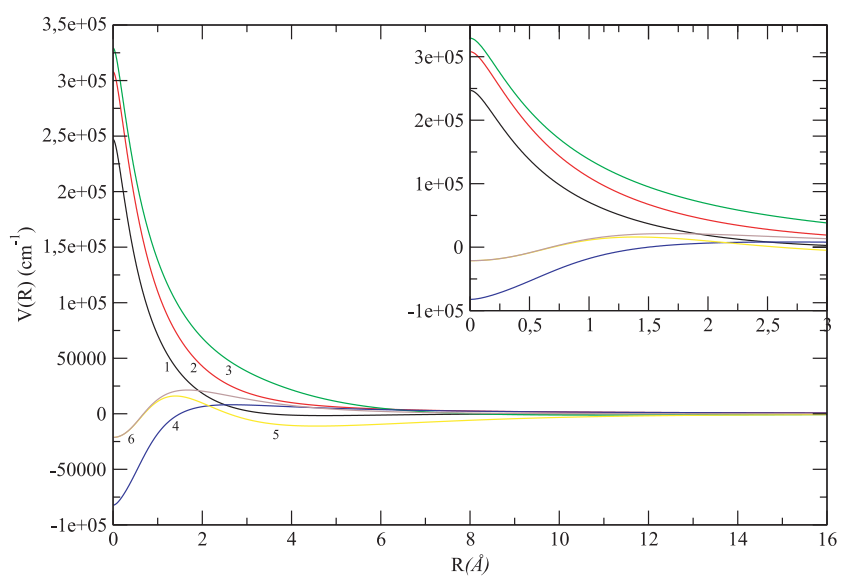

Figure 4. Transition difference potential as a function of the interatomic distance of $\mathrm{H}_{2}^{+}$of $\mathrm{Ly} \alpha$.

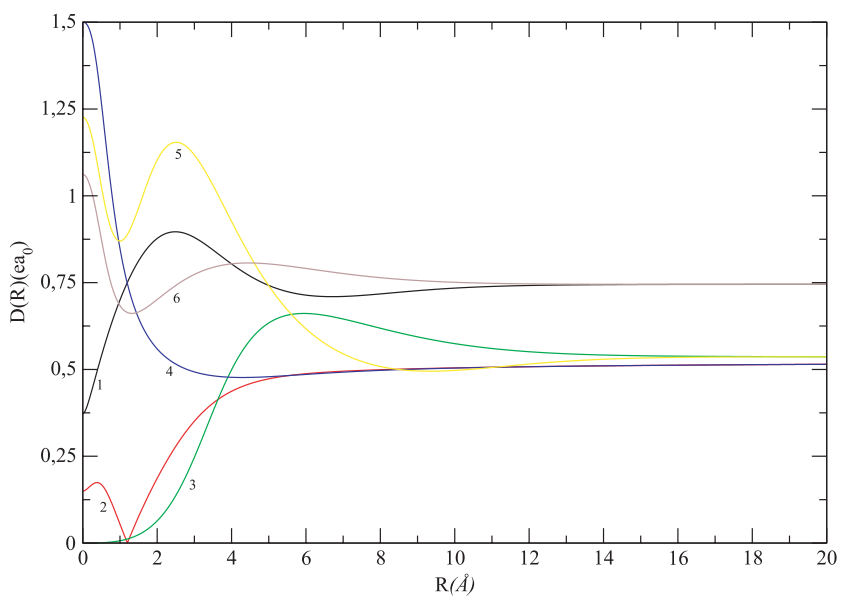

Figure 5. Electronic transition dipole moments of Ly $\alpha$ due to $\mathrm{H}$ perturbed by collisions with protons. $D(R)$ is given here in atomic units.

\subsubsection{Lyman $\beta$}

The profile of $\operatorname{Ly} \beta$ depends on 10 transitions. Table 3 lists all the wavelengths of these transitions and the possible satellite lines. Satellites of $\operatorname{Ly} \beta$ were observed in the spectra of the DA WD Wolf 1346 with the Hopkins Ultraviolet Telescope (HUT; Koester et al. 1996). Koester et al. (1998) observed satellites absorption features 
Table 2. Allowed transitions and satellites due to $\mathrm{H}-\mathrm{H}^{+}$collisions of $\mathrm{Ly} \alpha$ to the distance $R(\AA)$ of the atom-ion pair at which the potential extreme occurs.

\begin{tabular}{lccccc}
\hline $\begin{array}{l}\text { Label } \\
\text { level }\end{array}$ & $\begin{array}{c}\text { Upper } \\
\text { level }\end{array}$ & $\begin{array}{c}\Delta v_{\mathrm{H}_{2}^{+}} \\
\left(\mathrm{cm}^{-1}\right)\end{array}$ & $\begin{array}{c}\lambda_{\mathrm{H}_{2}^{+}} \\
(\AA)\end{array}$ & $\begin{array}{c}R \\
(\AA)\end{array}$ \\
\hline 1 & $2 p \pi_{\mathrm{u}}$ & $1 s \sigma_{\mathrm{g}}$ & -1628.54 & 1240.56 & 4.72 \\
2 & $3 p \sigma_{\mathrm{u}}$ & $1 s \sigma_{\mathrm{g}}$ & - & - & - \\
3 & $4 f \sigma_{\mathrm{u}}$ & $1 s \sigma_{\mathrm{g}}$ & -1172.40 & 1233.58 & 11.07 \\
4 & $2 s \sigma_{\mathrm{g}}$ & $2 p \sigma_{\mathrm{u}}$ & 8139.91 & 1106.47 & 2.65 \\
5 & $3 d \sigma_{\mathrm{g}}$ & $2 p \sigma_{\mathrm{u}}$ & -11055.90 & 1404.87 & 4.56 \\
& & & 16001.33 & 1017.93 & 1.39 \\
6 & $3 d \pi_{\mathrm{g}}$ & $2 p \sigma_{\mathrm{u}}$ & 21384.28 & 965.05 & 1.67 \\
\hline
\end{tabular}

Table 3. Allowed transitions and satellites due to $\mathrm{H}-\mathrm{H}^{+}$collisions of $\mathrm{Ly} \beta$ to the distance $R(\AA)$ of the atom-ion pair at which the potential extreme occurs.

\begin{tabular}{lccccc}
\hline Label & $\begin{array}{c}\text { Upper } \\
\text { level }\end{array}$ & $\begin{array}{c}\text { Lower } \\
\text { level }\end{array}$ & $\begin{array}{c}\Delta v_{\mathrm{H}_{2}^{+}} \\
\left(\mathrm{cm}^{-1}\right)\end{array}$ & $\begin{array}{c}\lambda_{\mathrm{H}_{2}^{+}} \\
(\AA)\end{array}$ & $\begin{array}{c}R \\
(\AA)\end{array}$ \\
\hline 1 & $3 p \pi_{\mathrm{u}}$ & $1 s \sigma_{\mathrm{g}}$ & - & - & - \\
2 & $4 p \sigma_{\mathrm{u}}$ & $1 s \sigma_{\mathrm{g}}$ & - & - & - \\
3 & $4 f \pi_{\mathrm{u}}$ & $1 s \sigma_{\mathrm{g}}$ & -3361.25 & 1062.64 & 9.85 \\
4 & $5 f \sigma_{\mathrm{u}}$ & $1 s \sigma_{\mathrm{g}}$ & - & - & - \\
5 & $6 h \sigma_{\mathrm{u}}$ & $1 s \sigma_{\mathrm{g}}$ & -1037.33 & 1037.03 & 21.45 \\
6 & $3 s \sigma_{\mathrm{g}}$ & $2 p \sigma_{\mathrm{u}}$ & 22200.04 & 835.65 & 1.96 \\
7 & $4 d \sigma_{\mathrm{g}}$ & $2 p \sigma_{\mathrm{u}}$ & -633.34 & 1032.71 & 9.46 \\
& & & 25868.59 & 810.80 & 1.55 \\
8 & $4 d \pi_{\mathrm{g}}$ & $2 p \sigma_{\mathrm{u}}$ & 28666.11 & 792.82 & 1.70 \\
9 & $5 g \sigma_{\mathrm{g}}$ & $2 p \sigma_{\mathrm{u}}$ & -4899.9272 & 1080.31 & 12.65 \\
& & & 38880.59 & 733.42 & 1.69 \\
10 & $5 g \pi_{\mathrm{g}}$ & $2 p \sigma_{\mathrm{u}}$ & -516.61 & 1031.46 & 18.89 \\
& & & 38914.86 & 733.24 & 1.69 \\
\hline
\end{tabular}

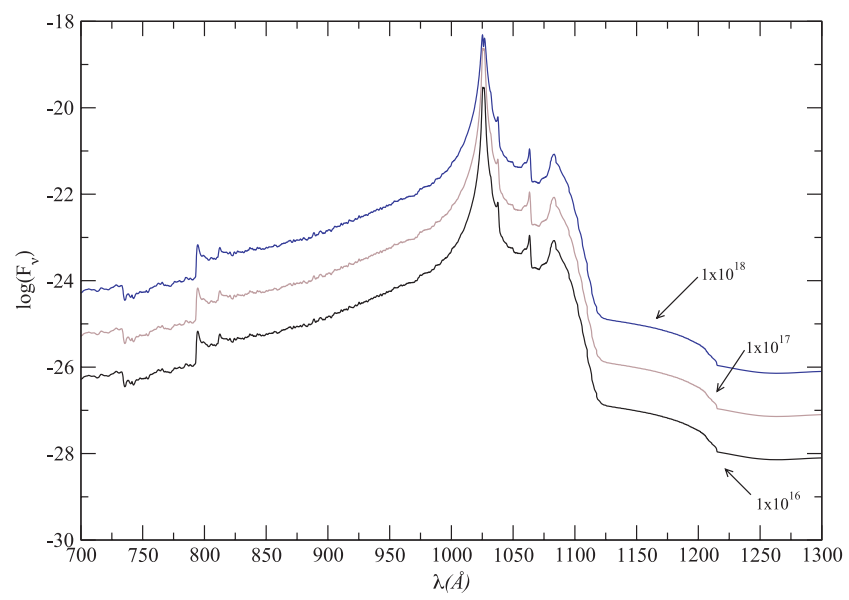

Figure 6. Ly $\beta$ profile for $T=4000 \mathrm{~K}$ and densities of $\mathrm{H}^{+}$perturbers of $n_{\mathrm{H}}^{+}=10^{16}, 10^{17}$ and $10^{18} \mathrm{~cm}^{-3}$ (from bottom to top).

at $\lambda=1060$ and $1080 \AA$ in the spectra of four other targets obtained with the Orbiting and Retrievable Far and Extreme Ultraviolet Spectrograph (ORFEUS). These satellites are due to the contributions of the transitions $4 f \pi_{\mathrm{u}}-1 s \sigma_{\mathrm{g}}$ and $5 g \sigma_{\mathrm{g}}-2 p \sigma_{\mathrm{u}}$ of the profile of Ly $\beta$, according to the profiles of calculated by Allard et al. (1998a).

The Ly $\beta$ line profiles shown in Figs 6 and 7 are for temperatures of 4000 and $12000 \mathrm{~K}$ and proton densities of $10^{16}, 10^{17}$ and

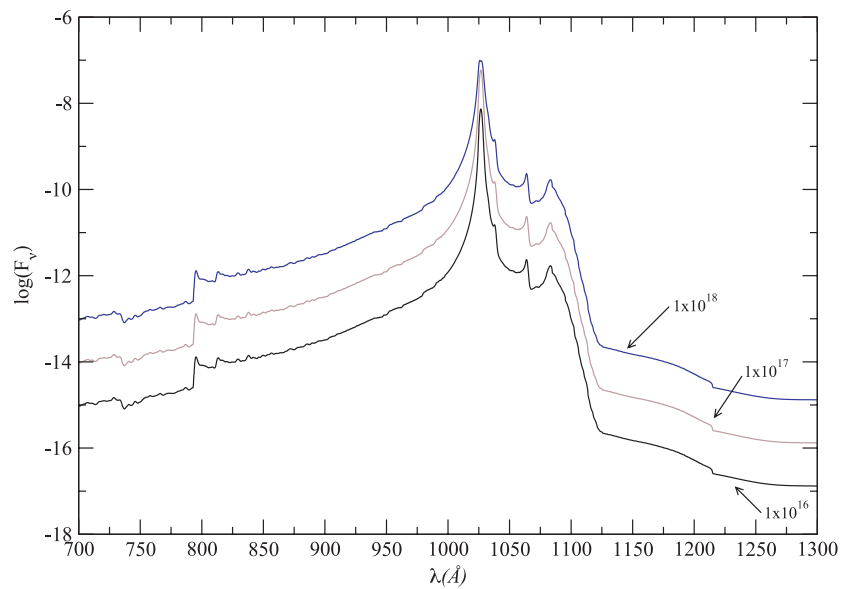

Figure 7. Ly $\beta$ profile for $T=12000 \mathrm{~K}$ and density of $\mathrm{H}^{+}$perturbers of $n_{\mathrm{H}}^{+}=10^{16}, 10^{17}$ and $10^{18} \mathrm{~cm}^{-3}$ (from bottom to top).

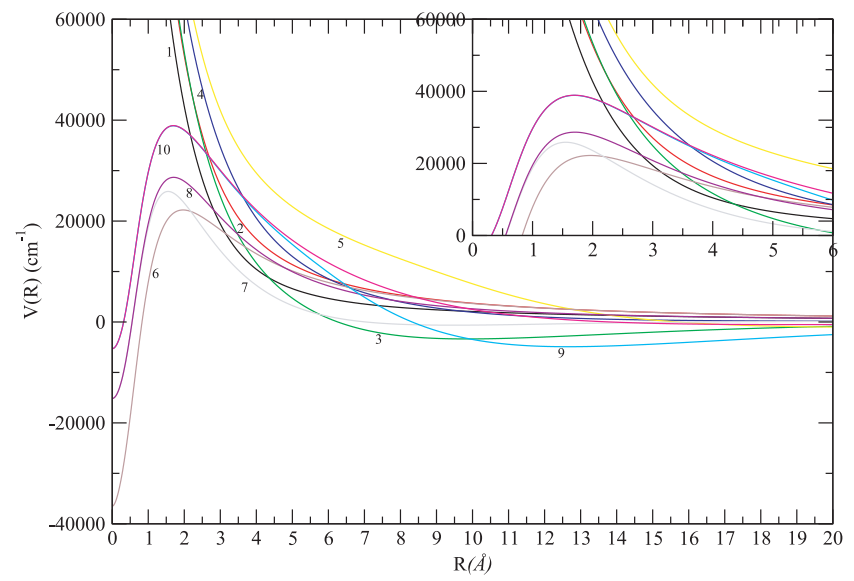

Figure 8. Transition potential energies as a function of the interatomic distance of $\mathrm{H}_{2}^{+}$of $\mathrm{Ly} \beta$.

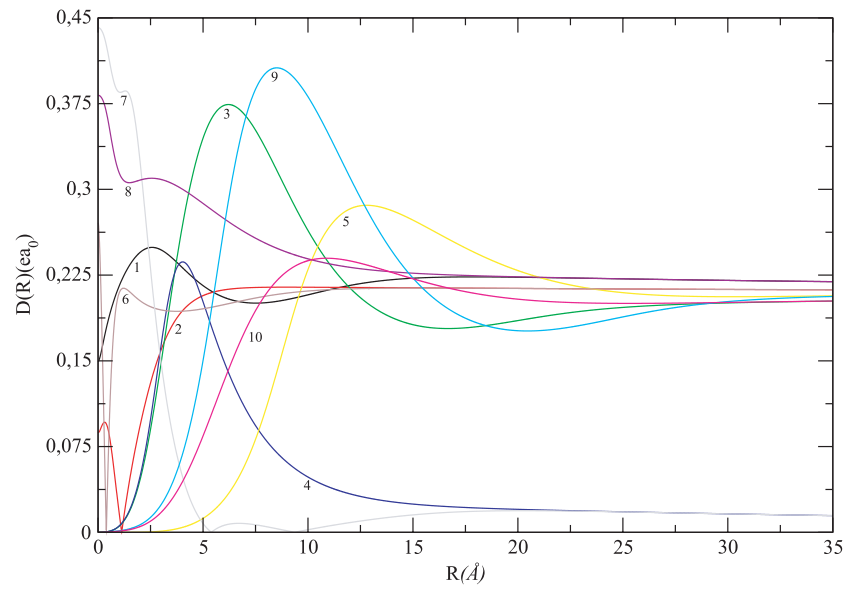

Figure 9. Electronic transition dipole moments of $\operatorname{Ly} \beta$ due to $\mathrm{H}$ perturbed by collisions with protons. $D(R)$ is given here in atomic units.

$10^{18} \mathrm{~cm}^{-3}$. Fig. 8 shows all the differences of the potential energy of the transitions that contribute to the $\operatorname{Ly} \beta$ line profile. In Fig. 9, we plot the dipole $D(R)$ for transitions of the $\operatorname{Ly} \beta$ due to $\mathrm{H}$ perturbed by collisions with protons. 


\subsubsection{Lyman $\gamma$}

There are 14 allowed transitions due to collisions of $\mathrm{H}-\mathrm{H}^{+}$and the satellites predicted to occur are listed in Table 4. The satellite absorption features at $\lambda=995 \AA$ was observed in a $H U T$ spectrum and later in spectra recorded by ORFEUS. This Ly $\gamma$ satellite was also visible in the spectra of the WD CD-38 10980 (Wolff et al. 2001) and Sirius B (Holberg et al. 2003). Allard et al. (2004) calculated the profiles of $\operatorname{Ly} \gamma$ line based on accurate $a b$ initio potentials to explain the observation of this feature.

The line profile calculations shown in Figs 10 and 11 have been evaluated at a temperature of 4000 and $12000 \mathrm{~K}$ for different densities of protons $10^{16}, 10^{17}$ and $10^{18} \mathrm{~cm}^{-3}$.

Fig. 12 shows all the potential energy differences for the transitions that contribute to the $\mathrm{Ly} \gamma$ line profile and Fig. 13 plots the electronic transition dipole moments for $\mathrm{Ly} \gamma$ due to $\mathrm{H}$ perturbed by collisions with protons.

Table 4. Allowed transitions and satellites due to $\mathrm{H}-\mathrm{H}^{+}$collisions of $\mathrm{Ly} \gamma$ to the distance $R(\AA)$ of the atom-ion pair at which the potential extreme occurs.

\begin{tabular}{lccccc}
\hline Label & $\begin{array}{c}\text { Upper } \\
\text { level }\end{array}$ & $\begin{array}{c}\text { Lower } \\
\text { level }\end{array}$ & $\begin{array}{c}\Delta v_{\mathrm{H}_{2}^{+}} \\
\left(\mathrm{cm}^{-1}\right)\end{array}$ & $\begin{array}{c}\lambda_{\mathrm{H}_{2}^{+}} \\
(\AA)\end{array}$ & $\begin{array}{c}R \\
(\AA)\end{array}$ \\
\hline 1 & $4 p \pi_{\mathrm{u}}$ & $1 s \sigma_{\mathrm{g}}$ & - & - & - \\
2 & $5 p \sigma_{\mathrm{u}}$ & $1 s \sigma_{\mathrm{g}}$ & - & - & - \\
3 & $5 f \pi_{\mathrm{u}}$ & $1 s \sigma_{\mathrm{g}}$ & -192.78 & 974.62 & 16.65 \\
4 & $6 f \sigma_{\mathrm{u}}$ & $1 s \sigma_{\mathrm{g}}$ & - & - & - \\
5 & $6 h \pi_{\mathrm{u}}$ & $1 s \sigma_{\mathrm{g}}$ & -2188.78 & 993.96 & 20.69 \\
6 & $7 h \sigma_{\mathrm{u}}$ & $1 s \sigma_{\mathrm{g}}$ & -230.90 & 974.98 & 29.66 \\
7 & $8 j \sigma_{\mathrm{u}}$ & $1 s \sigma_{\mathrm{g}}$ & -784.17 & 980.27 & 36.06 \\
8 & $4 s \sigma_{\mathrm{g}}$ & $2 p \sigma_{\mathrm{u}}$ & 30153.49 & 752.16 & 1.82 \\
9 & $5 d \sigma_{\mathrm{g}}$ & $2 p \sigma_{\mathrm{u}}$ & 32004.30 & 741.83 & 1.62 \\
10 & $5 d \pi_{\mathrm{g}}$ & $2 p \sigma_{\mathrm{u}}$ & 33520.98 & 733.58 & 1.70 \\
11 & $6 g \sigma_{\mathrm{g}}$ & $2 p \sigma_{\mathrm{u}}$ & -1395.38 & 986.18 & 17.79 \\
& & & 39005.56 & 705.21 & 1.69 \\
12 & $6 g \pi_{\mathrm{g}}$ & $2 p \sigma_{\mathrm{u}}$ & 39025.78 & 705.11 & 1.69 \\
13 & $7 i \sigma_{\mathrm{g}}$ & $2 p \sigma_{\mathrm{u}}$ & -2627.07 & 998.31 & 25.06 \\
& & & 42340.74 & 689.00 & 1.70 \\
14 & $7 i \pi_{\mathrm{g}}$ & $2 p \sigma_{\mathrm{u}}$ & -565.52 & 978.18 & 31.65 \\
& & & 42342.54 & 688.99 & 1.70 \\
\hline
\end{tabular}

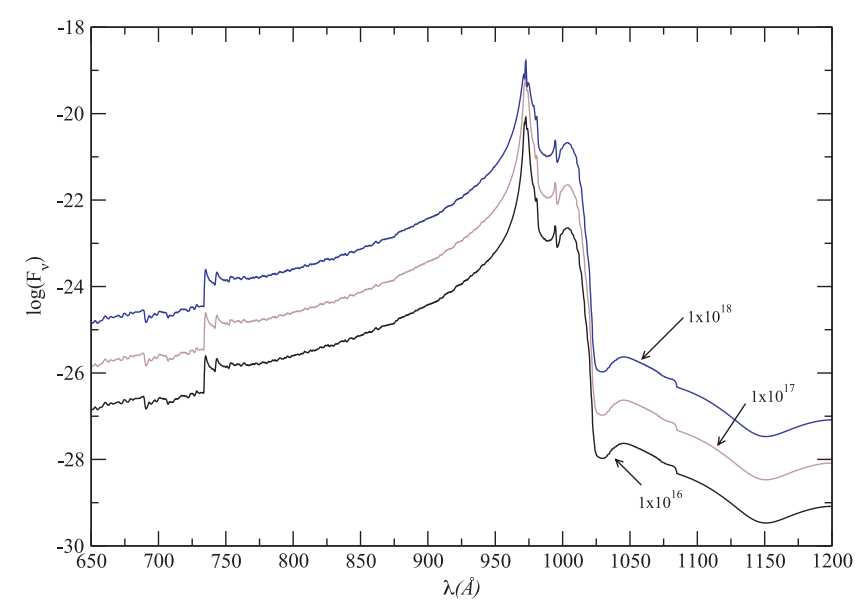

Figure 10. Variation of the $\mathrm{Ly} \gamma$ profile with density of $\mathrm{H}^{+}$perturbers $(T=4000 \mathrm{~K}), n_{\mathrm{H}}^{+}=10^{16}, 10^{17}$ and $10^{18} \mathrm{~cm}^{-3}$ (from bottom to top).

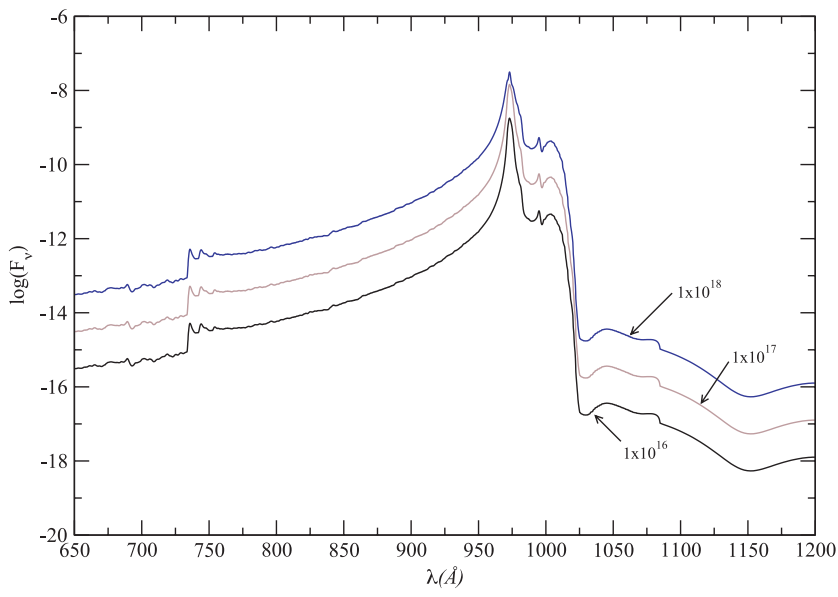

Figure 11. Variation of the $\mathrm{Ly} \gamma$ profile with density of $\mathrm{H}^{+}$perturbers $(T=12000 \mathrm{~K}), n_{\mathrm{H}}^{+}=10^{16}, 10^{17}$ and $10^{18} \mathrm{~cm}^{-3}$ (from bottom to top).

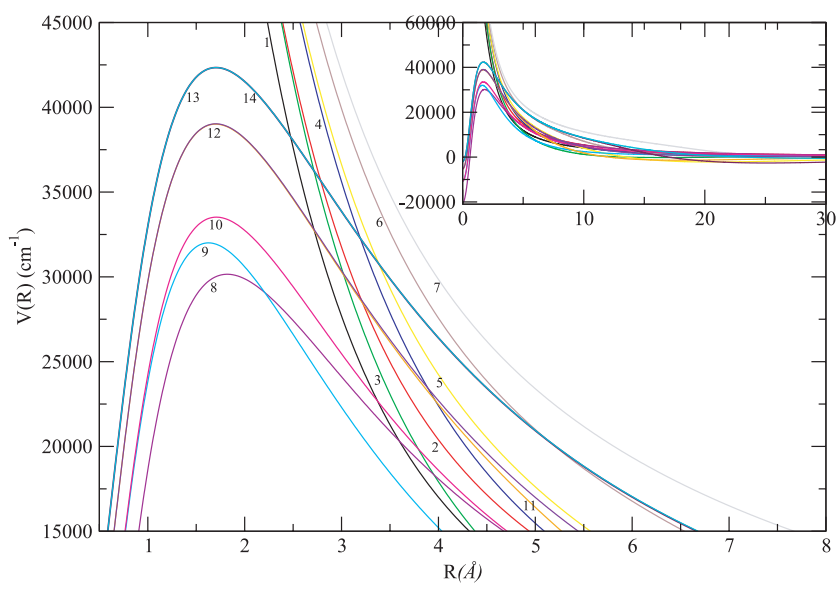

Figure 12. Transition potential energy differences as a function of the interatomic distance of $\mathrm{H}_{2}^{+}$of $\mathrm{Ly} \gamma$.

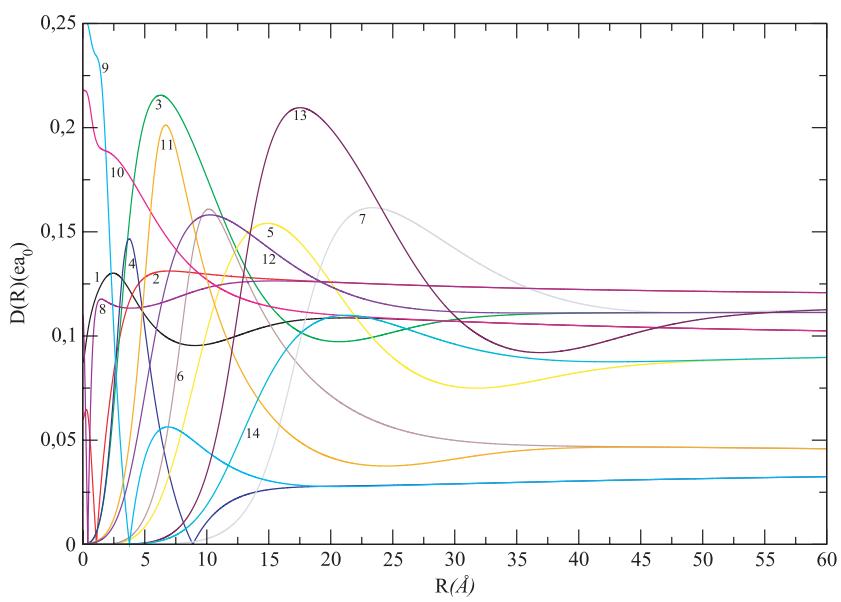

Figure 13. Electronic transition dipole moments of $\mathrm{Ly} \gamma$ due to $\mathrm{H}$ perturbed by collisions with protons. $D(R)$ is given here in atomic units.

\subsubsection{Lyman $\delta$}

There are 18 allowed transitions due to collisions of $\mathrm{H}_{\text {and }} \mathrm{H}^{+}$, and the satellites predicted to occur are listed in Table 5.

Figs 14 and 15 show the line profiles as a function of temperature and density of perturbers. 
Table 5. Allowed transitions and satellites due to $\mathrm{H}-\mathrm{H}^{+}$collisions of $\mathrm{Ly} \delta$ to the distance $R(\AA)$ of the atom-ion pair at which the potential extreme occurs.

\begin{tabular}{lccccc}
\hline Label & $\begin{array}{c}\text { Upper } \\
\text { level }\end{array}$ & $\begin{array}{c}\text { Lower } \\
\text { level }\end{array}$ & $\begin{array}{c}\Delta v_{\mathrm{H}_{2}^{+}} \\
\left(\mathrm{cm}^{-1}\right)\end{array}$ & $\begin{array}{c}\lambda_{\mathrm{H}^{+}} \\
(\AA)\end{array}$ & $\begin{array}{c}R \\
(\AA)\end{array}$ \\
\hline 1 & $5 p \pi_{\mathrm{u}}$ & $1 s \sigma_{\mathrm{g}}$ & - & - & - \\
2 & $6 p \sigma_{\mathrm{u}}$ & $1 s \sigma_{\mathrm{g}}$ & - & - & - \\
3 & $6 f \pi_{\mathrm{u}}$ & $1 s \sigma_{\mathrm{g}}$ & - & - & - \\
4 & $7 f \sigma_{\mathrm{u}}$ & $1 s \sigma_{\mathrm{g}}$ & - & - & - \\
5 & $7 h \pi_{\mathrm{u}}$ & $1 s \sigma_{\mathrm{g}}$ & -679.23 & 956.16 & 27.56 \\
6 & $8 h \sigma_{\mathrm{u}}$ & $1 s \sigma_{\mathrm{g}}$ & - & - & - \\
7 & $8 j \pi_{\mathrm{u}}$ & $1 s \sigma_{\mathrm{g}}$ & -1413.57 & 962.93 & 36.17 \\
8 & $9 j \sigma_{\mathrm{u}}$ & $1 s \sigma_{\mathrm{g}}$ & -312.56 & 952.82 & 44.76 \\
9 & $10 l \sigma_{\mathrm{u}}$ & $1 s \sigma_{\mathrm{g}}$ & -582.87 & 955.28 & 54.98 \\
10 & $5 s \sigma_{\mathrm{g}}$ & $2 p \sigma_{\mathrm{u}}$ & - & - & - \\
11 & $6 d \sigma_{\mathrm{g}}$ & $2 p \sigma_{\mathrm{u}}$ & - & - & - \\
12 & $6 d \pi_{\mathrm{g}}$ & $2 p \sigma_{\mathrm{u}}$ & - & - & - \\
13 & $7 g \sigma_{\mathrm{g}}$ & $2 p \sigma_{\mathrm{u}}$ & -76.95 & 950.69 & 26.02 \\
14 & $7 g \pi_{\mathrm{g}}$ & $2 p \sigma_{\mathrm{u}}$ & - & - & - \\
15 & $8 i \sigma_{\mathrm{g}}$ & $2 p \sigma_{\mathrm{u}}$ & -1119.44 & 960.21 & 31.54 \\
16 & $8 i \pi_{\mathrm{g}}$ & $2 p \sigma_{\mathrm{u}}$ & -99.84 & 950.90 & 42.38 \\
17 & $9 k \sigma_{\mathrm{g}}$ & $2 p \sigma_{\mathrm{u}}$ & -1589.40 & 964.56 & 41.91 \\
18 & $9 k \pi_{\mathrm{g}}$ & $2 p \sigma_{\mathrm{u}}$ & -475.45 & 954.31 & 49.00 \\
\hline
\end{tabular}

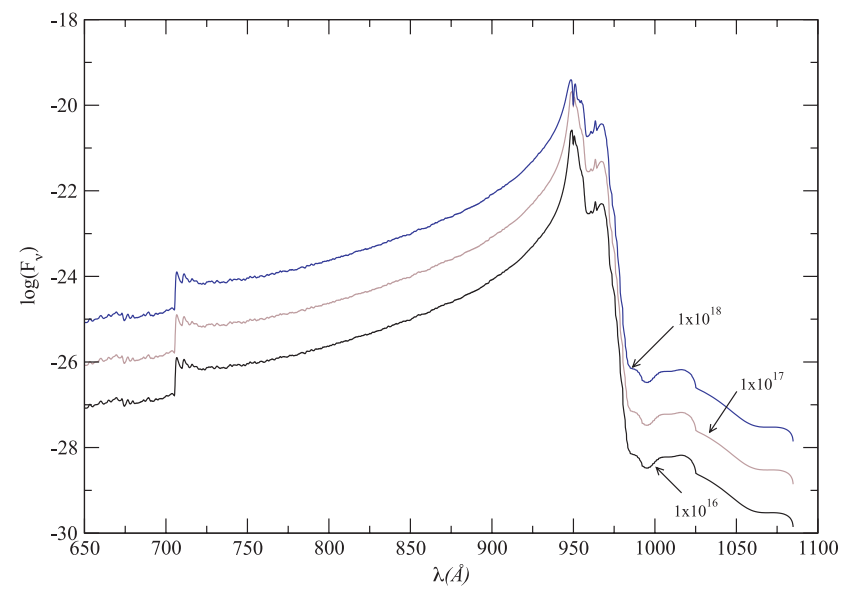

Figure 14. $\mathrm{Ly} \delta$ profile for density of $\mathrm{H}^{+}$perturbers $(T=4000 \mathrm{~K}), n_{\mathrm{H}}^{+}=$ $10^{16}, 10^{17}$ and $10^{18} \mathrm{~cm}^{-3}$ (from bottom to top).

Fig. 16 shows all the potential energy differences for the transitions that contribute to the line profile of Ly $\delta$. Fig. 17 shows the electronic transition dipole moments for $\mathrm{Ly} \delta$ of the $\mathrm{H}_{2}^{+}$and Fig. 18 shows the total line profiles of $\operatorname{Ly} \alpha, \operatorname{Ly} \beta, \operatorname{Ly} \gamma$ and $\operatorname{Ly} \delta$ perturbed by protons for a single $T=12000 \mathrm{~K}$ and $n_{\mathrm{H}}^{+}=10^{17} \mathrm{~cm}^{-3}$. This figure is similar to Fig. 3 obtained by Hébrard et al. (2011), showing that our calculations and approximations are consistent with data already published.

\subsection{Balmer series}

The Balmer lines have transitions that must obey the same golden rules as the Lyman series transitions.

\subsubsection{Balmer $\alpha$}

The total profile of Balmer $\alpha$ depends on 32 individual transitions. The line profile calculations shown in Fig. 23 have been calculated at a single temperature of $12000 \mathrm{~K}$ and a proton density of $10^{17} \mathrm{~cm}^{-3}$.

Table 6 lists only the transitions that generate possible satellite lines on the wings of the Balmer line profiles. In Fig. 19 we plot

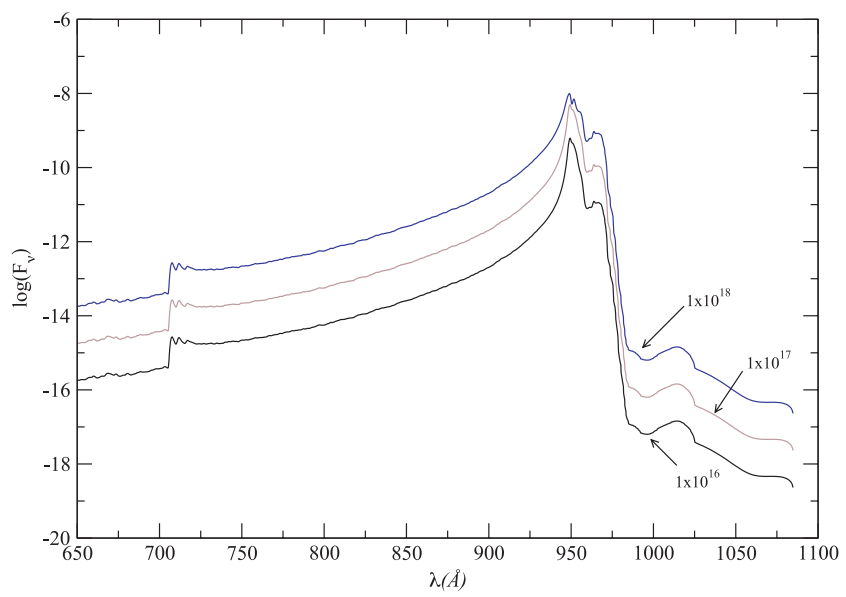

Figure 15. Ly $\delta$ profile for density of $\mathrm{H}^{+}$perturbers $(T=12000 \mathrm{~K}), n_{\mathrm{H}}^{+}=$ $10^{16}, 10^{17}$ and $10^{18} \mathrm{~cm}^{-3}$ (from bottom to top).

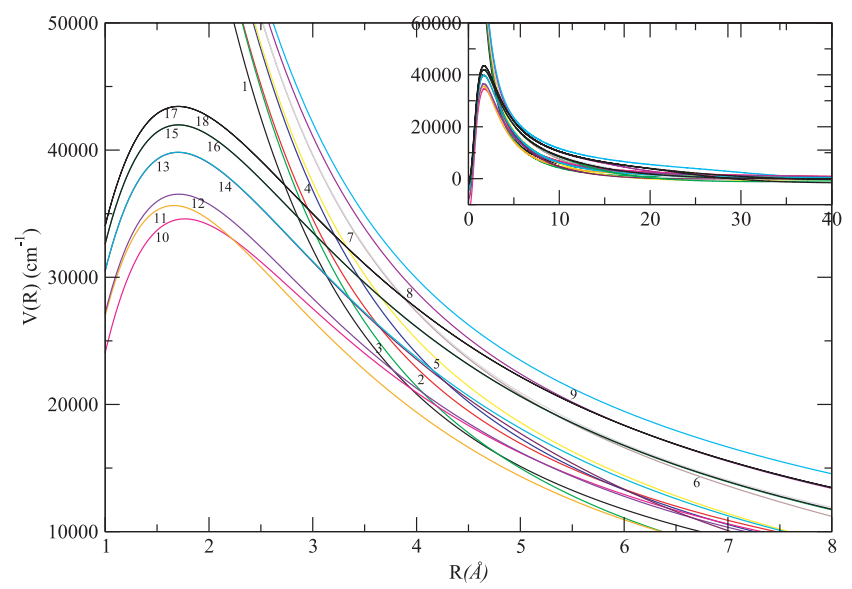

Figure 16. Potential energy differences for the allowed transitions, as a function of the interatomic distance of $\mathrm{H}_{2}^{+}$of $\mathrm{Ly} \delta$.

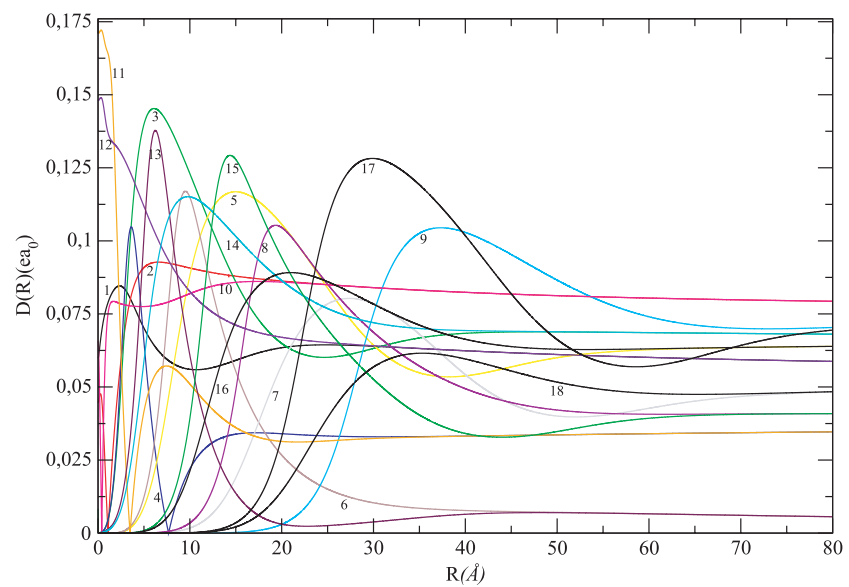

Figure 17. Electronic transition dipole moments of $\mathrm{Ly} \delta$ due to $\mathrm{H}$ perturbed by collisions with protons. $D(R)$ is given here in atomic units.

the transition dipole moments for the satellites listed in Table 6. In laboratory observations, measurements of the spectrum of a laserproduced plasma confirm that the $\lambda=8650 \AA$ satellite exists in the $\mathrm{H} \alpha$ red wing (Kielkopf et al. 2002) due the transitions $5 g \sigma_{\mathrm{g}}-4 f \sigma_{\mathrm{u}}$ and $4 f \pi_{\mathrm{u}}-3 d \pi_{\mathrm{g}}$. 


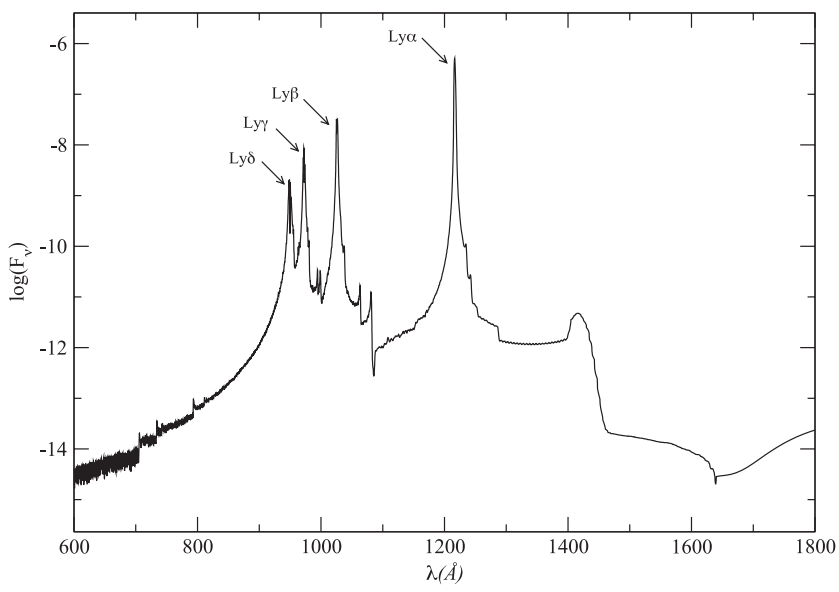

Figure 18. Total profile of $\operatorname{Ly} \alpha, \operatorname{Ly} \beta, \operatorname{Ly} \gamma$ and $\operatorname{Ly} \delta$ perturbed by protons $\left(n_{\mathrm{H}}^{+}=10^{17} \mathrm{~cm}^{-3}\right)$ at a temperature of $T=12000 \mathrm{~K}$.

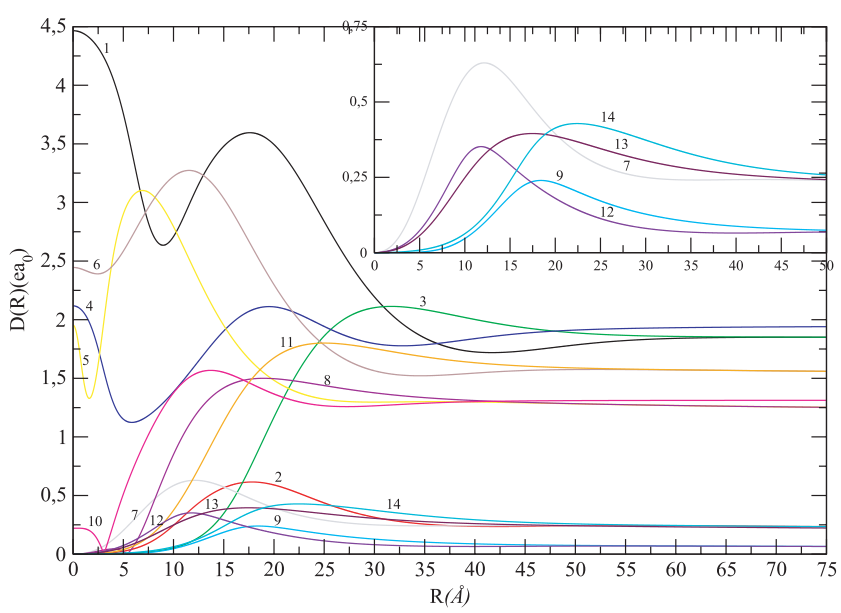

Figure 19. Transition dipole moments for the satellites of Balmer $\alpha$ due to $\mathrm{H}$ perturbed by collision with protons. The transitions plotted here are listed in Table 6. $D(R)$ is given here in atomic units.

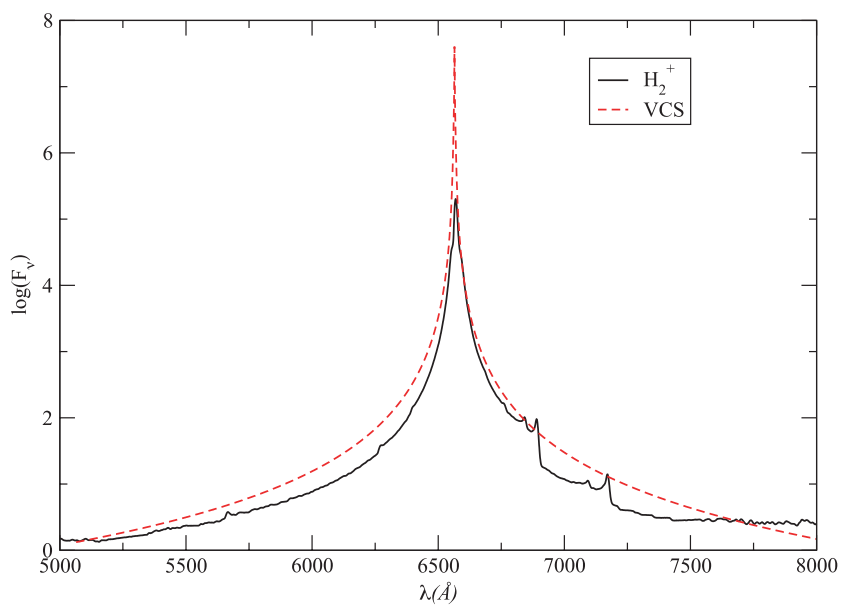

Figure 20. Comparison between the profile of Balmer $\alpha$ of $\mathrm{H}_{2}^{+}$with the VCS profile - electron Stark broadening, for $T=12000 \mathrm{~K}$ and density $10^{16} \mathrm{~cm}^{-3}$.

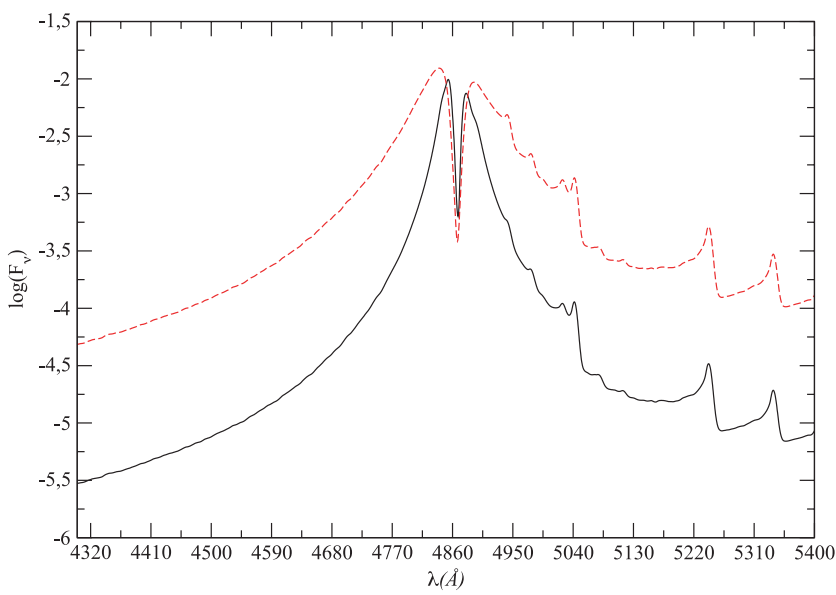

Figure 21. Solid line denotes the Balmer $\beta$ profile with density $n_{\mathrm{H}}^{+}=$ $9.324 \times 10^{16} \mathrm{~cm}^{-3}$ of the perturbers and $T=13526 \mathrm{~K}$. Dashed line denotes the Balmer $\beta$ profile with density $n_{\mathrm{H}}^{+}=1.554 \times 10^{18} \mathrm{~cm}^{-3}$ of the perturbers and $T=13526 \mathrm{~K}$. The line profile can be used for diagnostic of the physical conditions of a laboratory measurement.

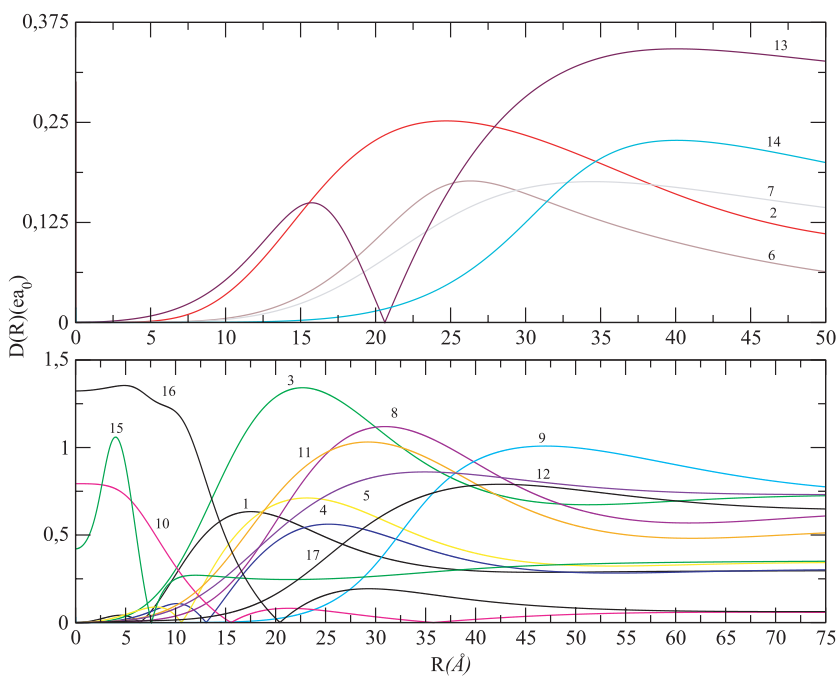

Figure 22. Electronic transition dipole moments for the satellites of Balmer $\beta$ due to $\mathrm{H}$ perturbed by collision with protons. The transitions plotted here are listed in Table 7. $D(R)$ is given in atomic units.

Fig. 20 shows the comparison between the VCS (Vidal, Cooper \& Smith 1973) profile calculated by Koester (private communication) for $T=12000 \mathrm{~K}$ and $n_{\mathrm{H}}^{+}$density $10^{16} \mathrm{~cm}^{-3}$, with the profile of $\mathrm{H}_{2}^{+}$ calculated for the same temperature and density. It is observed that at high energies both profiles coincide asymptotically, while, due to possible formation of low-energy molecules, discrepancies arise between the profiles.

\subsubsection{Balmer $\beta$}

The total profile of Balmer $\beta$ depends on 46 individual transitions. Fig. 21 shows the profile of $\mathrm{H} \beta$ calculated for proton densities of $9.324 \times 10^{16} \mathrm{~cm}^{-3}$ and $1.554 \times 10^{18} \mathrm{~cm}^{-3}$ with $T=13526 \mathrm{~K}$. We can see that the centre of the line is split and asymmetric.

Table 7 lists the satellite lines due to $\mathrm{H} \beta$ and Fig. 22 plots the respective transitions listed in this table. The line profile calculations shown in Fig. 23 are for a temperature of $12000 \mathrm{~K}$ and proton density of $10^{17} \mathrm{~cm}^{-3}$. 

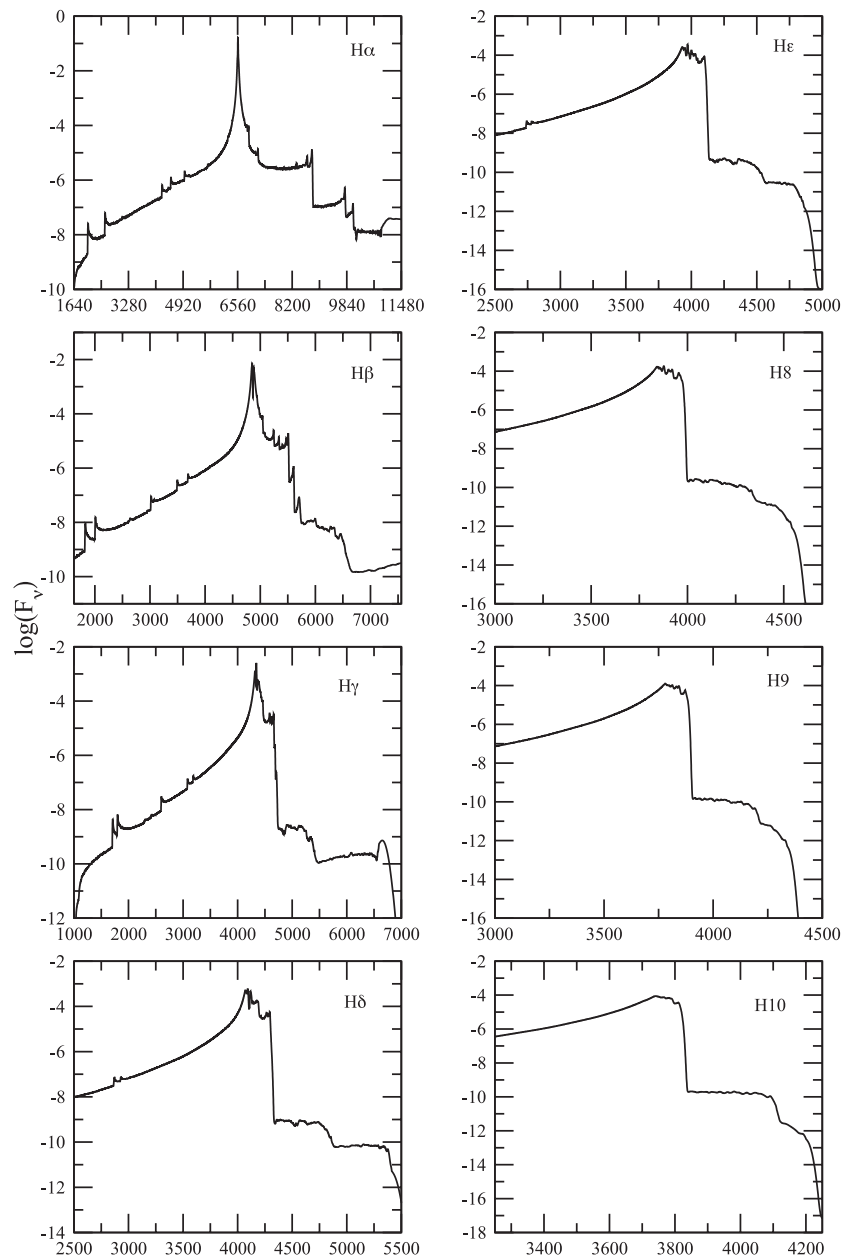

$\lambda(A)$

Figure 23. Profiles of the Balmer series $(\mathrm{H} \alpha, \mathrm{H} \beta, \mathrm{H} \gamma, \mathrm{H} \delta, \mathrm{H} \epsilon, \mathrm{H} 8, \mathrm{H} 9$ and H10) for ion density $n_{\mathrm{H}}^{+}=10^{17} \mathrm{~cm}^{-3}$ and $T=12000 \mathrm{~K}$.

\subsubsection{Balmer $\gamma$}

The total profile of Balmer $\gamma$ depends on 60 individual transitions. The line profile shown in Fig. 23 is for a temperature of $12000 \mathrm{~K}$ and proton density $10^{17} \mathrm{~cm}^{-3}$.

\subsubsection{Balmer $\delta$}

The total profile of Balmer $\delta$ depends on 74 individual transitions. The line profile calculations of Fig. 23 have been carried out at a temperature of $12000 \mathrm{~K}$ for the proton density $10^{17} \mathrm{~cm}^{-3}$.

\subsubsection{Balmer $\epsilon$}

The total profile of Balmer $\epsilon$ depends on 88 individual transitions. The line profile calculations of Fig. 23 have been carried out at a temperature of $12000 \mathrm{~K}$ for the proton density $10^{17} \mathrm{~cm}^{-3}$.

\subsubsection{Balmer 8}

The total profile of Balmer 8 depends on 102 individual transitions. The line profile calculations of Fig. 23 have been carried out at a temperature of $12000 \mathrm{~K}$ for the proton density $10^{17} \mathrm{~cm}^{-3}$.
Table 6. Satellites due to $\mathrm{H}-\mathrm{H}^{+}$collisions of Balmer $\alpha$ to the distance $R$ ( $\AA)$ of the atom-ion pair at which the potential extreme occurs.

\begin{tabular}{|c|c|c|c|c|c|}
\hline Label & $\begin{array}{l}\text { Upper } \\
\text { level }\end{array}$ & $\begin{array}{c}\text { Lower } \\
\text { level }\end{array}$ & $\begin{array}{l}\Delta v_{\mathrm{H}_{2}^{+}} \\
\left(\mathrm{cm}^{-1}\right)\end{array}$ & $\begin{array}{l}\lambda_{\mathrm{H}_{2}^{+}} \\
(\AA)\end{array}$ & $\begin{array}{c}R \\
(\AA)\end{array}$ \\
\hline 1 & $5 g \sigma_{\mathrm{g}}$ & $4 f \sigma_{\mathrm{u}}$ & $\begin{array}{c}-3844.18 \\
4956.58\end{array}$ & $\begin{array}{l}8783.59 \\
4954.01\end{array}$ & $\begin{array}{c}13.10 \\
5.60\end{array}$ \\
\hline 2 & $5 g \sigma_{\mathrm{g}}$ & $2 p \pi_{\mathrm{u}}$ & -4996.57 & 9772.81 & 12.66 \\
\hline 3 & $6 h \sigma_{\mathrm{u}}$ & $3 d \sigma_{\mathrm{g}}$ & $\begin{array}{c}40925.21 \\
-702.54\end{array}$ & $\begin{array}{l}1780.80 \\
6883.97\end{array}$ & $\begin{array}{c}3.17 \\
23.03\end{array}$ \\
\hline 4 & $4 f \pi_{\mathrm{u}}$ & $3 d \sigma_{\mathrm{g}}$ & $\begin{array}{l}-1271.27 \\
23797.73\end{array}$ & $\begin{array}{l}7164.46 \\
2562.34\end{array}$ & $\begin{array}{c}13.13 \\
3.06\end{array}$ \\
\hline 5 & $4 d \sigma_{\mathrm{g}}$ & $3 p \sigma_{\mathrm{u}}$ & $\begin{array}{l}-3214.16 \\
12145.77\end{array}$ & $\begin{array}{l}8323.01 \\
3652.99\end{array}$ & $\begin{array}{l}6.47 \\
1.01\end{array}$ \\
\hline 6 & $4 f \pi_{\mathrm{u}}$ & $3 d \pi_{\mathrm{g}}$ & $\begin{array}{c}-3658.85 \\
7163.74\end{array}$ & $\begin{array}{l}8642.89 \\
4465.72\end{array}$ & $\begin{array}{l}9.33 \\
1.69\end{array}$ \\
\hline 7 & $4 f \pi_{\mathrm{u}}$ & $2 s \sigma_{\mathrm{g}}$ & $\begin{array}{r}-5245.35 \\
37537.98\end{array}$ & $\begin{array}{c}10016.33 \\
1895.12\end{array}$ & $\begin{array}{l}8.69 \\
1.00\end{array}$ \\
\hline 8 & $5 f \sigma_{\mathrm{u}}$ & $2 s \sigma_{\mathrm{g}}$ & -424.84 & 6754.83 & 15.47 \\
\hline 9 & $6 h \sigma_{\mathrm{u}}$ & $2 s \sigma_{\mathrm{g}}$ & -1500.75 & 7284.22 & 20.33 \\
\hline 10 & $4 d \sigma_{\mathrm{g}}$ & $2 p \pi_{\mathrm{u}}$ & -634.82 & 6852.02 & 10.20 \\
\hline 11 & $5 g \pi_{\mathrm{g}}$ & $2 p \pi_{\mathrm{u}}$ & -601.70 & 6836.51 & 18.74 \\
\hline 12 & $5 g \sigma_{\mathrm{g}}$ & $3 p \sigma_{\mathrm{u}}$ & $\begin{array}{l}-6042.60 \\
23287.20\end{array}$ & $\begin{array}{c}10885.60 \\
2596.30\end{array}$ & $\begin{array}{c}12.08 \\
1.18\end{array}$ \\
\hline 13 & $5 g \pi_{g}$ & $3 p \sigma_{\mathrm{u}}$ & $\begin{array}{l}-1130.91 \\
23303.15\end{array}$ & $\begin{array}{l}7093.14 \\
2595.23\end{array}$ & $\begin{array}{c}16.58 \\
1.18\end{array}$ \\
\hline 14 & $6 h \sigma_{\mathrm{u}}$ & $3 d \pi_{\mathrm{g}}$ & $\begin{array}{l}-1117.22 \\
23163.48\end{array}$ & $\begin{array}{l}7086.25 \\
2604.67\end{array}$ & $\begin{array}{c}21.38 \\
2.10\end{array}$ \\
\hline
\end{tabular}

Table 7. Satellites due to $\mathrm{H}-\mathrm{H}^{+}$collisions of Balmer $\beta$ to the distance $R$ ( $⿱$ ) of the atom-ion pair at which the potential extreme occurs.

\begin{tabular}{|c|c|c|c|c|c|}
\hline Label & $\begin{array}{c}\text { Upper } \\
\text { level }\end{array}$ & $\begin{array}{c}\text { Lower } \\
\text { level }\end{array}$ & $\begin{array}{c}\Delta v_{\mathrm{H}_{2}^{+}} \\
\left(\mathrm{cm}^{-1}\right)\end{array}$ & $\begin{array}{l}\lambda_{\mathrm{H}_{2}^{+}} \\
(\AA)\end{array}$ & $\begin{array}{c}R \\
(\AA)\end{array}$ \\
\hline 1 & $5 f \pi_{\mathrm{u}}$ & $2 s \sigma_{\mathrm{g}}$ & -992.00 & 5110.59 & 13.83 \\
\hline 2 & $6 h \pi_{\mathrm{u}}$ & $2 s \sigma_{\mathrm{g}}$ & -2674.11 & 5591.24 & 19.90 \\
\hline 3 & $5 g \delta_{\mathrm{g}}$ & $2 p \pi_{\mathrm{u}}$ & -1466.46 & 5237.59 & 16.80 \\
\hline 4 & $6 g \sigma_{\mathrm{g}}$ & $2 p \pi_{\mathrm{u}}$ & -1483.16 & 5242.17 & 17.74 \\
\hline 5 & $6 g \sigma_{\mathrm{g}}^{\circ}$ & $3 p \sigma_{\mathrm{u}}$ & $\begin{array}{l}-2040.74 \\
23365.48\end{array}$ & $\begin{array}{l}5400.01 \\
2276.62\end{array}$ & $\begin{array}{c}16.50 \\
1.19\end{array}$ \\
\hline 6 & $7 i \sigma_{\mathrm{g}}$ & $3 p \sigma_{\mathrm{u}}$ & $\begin{array}{l}-2977.17 \\
26648.74\end{array}$ & $\begin{array}{l}5687.62 \\
2118.28\end{array}$ & $\begin{array}{c}24.59 \\
1.19\end{array}$ \\
\hline 7 & $7 i \pi_{\mathrm{g}}$ & $3 p \sigma_{\mathrm{u}}$ & $\begin{array}{c}-816.65 \\
26649.62\end{array}$ & $\begin{array}{l}5065.20 \\
2118.24\end{array}$ & $\begin{array}{c}29.99 \\
1.19\end{array}$ \\
\hline 8 & $6 h \pi_{\mathrm{u}}$ & $3 d \sigma_{\mathrm{g}}$ & $\begin{array}{l}-1811.98 \\
35621.24\end{array}$ & $\begin{array}{l}5334.12 \\
1779.97\end{array}$ & $\begin{array}{c}21.66 \\
3.17\end{array}$ \\
\hline 9 & $8 j \sigma_{\mathrm{u}}$ & $3 d \sigma_{\mathrm{g}}$ & $\begin{array}{c}-381.85 \\
41130.19\end{array}$ & $\begin{array}{l}4956.05 \\
1621.02\end{array}$ & $\begin{array}{c}29.99 \\
3.19\end{array}$ \\
\hline 10 & $5 f \pi_{\mathrm{u}}$ & $3 d \pi_{\mathrm{g}}$ & $\begin{array}{c}-285.48 \\
12088.84\end{array}$ & $\begin{array}{l}4932.49 \\
3062.96\end{array}$ & $\begin{array}{c}16.46 \\
1.91\end{array}$ \\
\hline 11 & $6 h \pi_{\mathrm{u}}$ & $3 d \pi_{\mathrm{g}}$ & $\begin{array}{l}-2269.96 \\
17843.96\end{array}$ & $\begin{array}{l}5467.69 \\
2603.95\end{array}$ & $\begin{array}{c}20.64 \\
2.11\end{array}$ \\
\hline 12 & $6 h \delta_{\mathrm{u}}$ & $3 d \pi_{\mathrm{g}}$ & $\begin{array}{c}-308.92 \\
17875.84\end{array}$ & $\begin{array}{l}4938.20 \\
2601.79\end{array}$ & $\begin{array}{c}28.53 \\
2.13\end{array}$ \\
\hline 13 & $7 h \sigma_{\mathrm{u}}$ & $3 d \pi_{\mathrm{g}}$ & $\begin{array}{c}-302.86 \\
21106.24\end{array}$ & $\begin{array}{l}4936.72 \\
2400.06\end{array}$ & $\begin{array}{c}29.57 \\
2.12\end{array}$ \\
\hline 14 & $8 j \sigma_{\mathrm{u}}$ & $3 d \pi_{\mathrm{g}}$ & $\begin{array}{l}-853.70 \\
23255.19\end{array}$ & $\begin{array}{l}5074.72 \\
2282.35\end{array}$ & $\begin{array}{c}36.03 \\
2.15\end{array}$ \\
\hline 15 & $5 d \sigma_{\mathrm{g}}$ & $4 f \sigma_{\mathrm{u}}$ & $\begin{array}{c}-11612.48 \\
3925.46\end{array}$ & $\begin{array}{c}11177.27 \\
4084.18\end{array}$ & $\begin{array}{l}1.95 \\
8.17\end{array}$ \\
\hline 16 & $6 g \sigma_{\mathrm{g}}$ & $4 f \sigma_{\mathrm{u}}$ & $\begin{array}{c}-878.51 \\
6602.41\end{array}$ & $\begin{array}{l}5081.12 \\
3681.66\end{array}$ & $\begin{array}{c}20.00 \\
6.08\end{array}$ \\
\hline 17 & $7 i \pi_{\mathrm{g}}$ & $4 f \sigma_{\mathrm{u}}$ & $\begin{array}{c}-405.68 \\
12418.51\end{array}$ & $\begin{array}{l}4961.91 \\
3032.34\end{array}$ & $\begin{array}{c}29.99 \\
6.81\end{array}$ \\
\hline
\end{tabular}




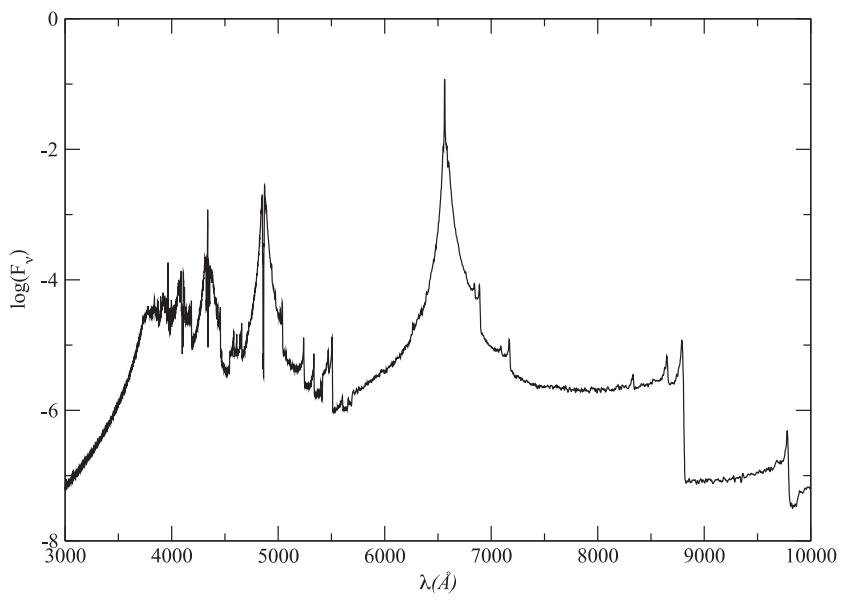

Figure 24. Total profile of the Balmer for $T=12000 \mathrm{~K}$ and $n_{\mathrm{H}}^{+}=$ $10^{17} \mathrm{~cm}^{-3}$.

\subsubsection{Balmer 9}

The total profile of Balmer 9 depends on 116 individual transitions. The line profile calculations of Fig. 23 have been carried out at a temperature of $12000 \mathrm{~K}$ for the proton density $10^{17} \mathrm{~cm}^{-3}$.

\subsubsection{Balmer 10}

The total profile of Balmer 10 depends on 130 individual transitions. The line profile calculations of Fig. 23 have been carried out at a temperature of $12000 \mathrm{~K}$ for the proton density $10^{17} \mathrm{~cm}^{-3}$.

\section{DISCUSSION AND CONCLUSION}

Satisfactory theory and data for the line profiles do exist due to the electron Stark broadening of neutral hydrogen (Vidal et al. 1973; Lemke 1997) and for 21 optical lines of neutral helium (Barnard, Cooper \& Shamey 1969; Beauchamp, Wesemael \& Bergeron 1997). The first three Lyman lines of $\mathrm{H}$ broadened by ionized and neutral perturbers, including a number of satellite features, are well described by Allard and collaborators (e.g. Allard et al. 2009 and references therein), and the atomic data are obtained from a number of atomic data bases, predominantly the line lists from Kurucz and collaborators (Kurucz \& Bell 1995), and the VALD (Vienna Atomic Line Database) data base (Piskunov et al. 1995; Ryabchikova et al. 1997; Kupka et al. 1999, 2000). For all other cases the situation is much less satisfactory, so there is a need for theories and data to explain the line profiles and satellite lines.

We extended the dipole potentials and line profile calculations to $n=10$ for the collision of $\mathrm{H}$ on $\mathrm{H}^{+}$perturbers important at high densities, as observed in the atmosphere of WD stars.

The laboratory observations of a laser-produced plasma confirm that satellites appear on $\operatorname{Ly} \alpha$ due to collisions with neutral atoms and protons. This experimental confirmation of the theory supports the identification of these features in the Ly $\alpha$ spectra of WD and $\lambda$ Bootis stars. In high temperature plasmas such as laser-produced plasmas, dielectronic recombination process is very important. This process strongly affects the ion abundances and produces dielectronic satellite lines. The satellite lines appear near the resonance line through spontaneous radiative decay from the autoionizing states. Satellite line emissions from highly ionized ions are often used for plasma diagnostics which are important in various kinds of plasmas, e.g. Tokamak, inertial fusion, astrophysical and plasma sources (Yamamoto, Kato \& Rosmej 2004). The comparison of the Ly $\alpha$ wing with line-shape models is a tool for determining neutral and proton densities in a hydrogenic plasma. These experiments also confirm that the variation of the radiative dipole moment is an important factor in determining the far-wing emission of Ly $\alpha$. When $D(R)$ differs significantly from its asymptotic value at an $R$ close to the region forming a satellite, the strength of the wing may be enhanced (or diminished) considerably.

In a collision-induced spectrum such as this one, the dipole moment may be more important than the potential in determining the shape of the satellite. These many-body perturbations alter the far line wing, adding multiple satellites and producing a strong continuum from the vacuum ultraviolet to the ultraviolet and visible.

The present calculations are carried out in an adiabatic approximation using a rectilinear trajectory. This should slightly affect the shape of the satellite, although no significant differences are expected.

The sum of the profiles calculated of $\operatorname{Ly} \alpha, \operatorname{Ly} \beta, \operatorname{Ly} \gamma$ and $\operatorname{Ly} \delta$ is shown in Fig. 18 and the sum of the profiles of Balmer series in Fig. 24 to $T=12000 \mathrm{~K}$ and $n_{\mathrm{H}}^{+}=10^{17} \mathrm{~cm}^{-3}$. The ion collision profiles differ significantly from the electron Stark broadening, showing not only satellite lines but also significant wing values that affect the opacity.

The data are available on http://astro.if.ufrgs.br/marcios.

\section{REFERENCES}

Allard N. F., Kielkopf J. F, 1982, Rev. Mod. Phys., 54, 1103

Allard N. F., Kielkopf J. F., Feautrier N., 1998a, A\&A, 330, 782

Allard N. F., Drira I., Gerbaldi M., Kielkopf J., Spielfiedel A., 1998b, A\&A, 335,1124

Allard N. F., Royer A., Kielkopf J. F., Feautrier N., 1999, Phys. Rev. A, 60, 1021

Allard N. F., Kielkopf J., Drira I., Schmelcher P., 2000, Eur. Phys. J. D, 12, 263

Allard N. F., Kielkopf J. F., Hébrard G., Peek J. M., 2004, Eur. Phys. J. D, 29,7

Allard N. F., Noselidze I., Kruk J. W., 2009, A\&A, 506, 993

Baber W. G., Hassé H. R., 1935, Proc. Camb. Phil. Soc., 31, 564

Barnard A. J., Cooper J., Shamey L. J., 1969, A\&A, 1, 28

Bates D. R., Poots G., 1953, Proc. Phys. Soc. A, 66, 784

Beauchamp A., Wesemael F., Bergeron P., 1997, ApJS, 108, 559

Bergeron P., Leggett S. K., 2002, ApJ, 580, 1070

Bergeron P., Wesemael F., Fontaine G., 1991, ApJ, 367, 253

Bergeron P., Saffer R. A., Liebert J., 1992, ApJ, 394, 228

Bergeron P., Wesemael F., Lamontagne R., Fontaine G., Saffer R. A., Allard N. F., 1995, ApJ, 449, 258

Burrau Ø., 1927, Naturwissenschaften, 15, 16

Eisenstein D. J. et al., 2006, ApJS, 167, 40

Engelbrecht A., Koester D., 2007, in Napiwotzki R., Barstow M., eds, ASP Conf. Ser. Vol. 372, 15th European Workshop on White Dwarfs. Astron. Soc. Pac., San Francisco, p. 289

Falcon R. E., Winget D. E., Montgomery M. H., Williams K. A., 2010, ApJ, 712,585

Frommhold L., 1993, Collision-induced Absorption in Gases. Cambridge Univ. Press, Cambridge

Gianninas A., Bergeron P., Dupuis J., Ruiz M. T., 2010, ApJ, 720, 581

Hébrard G., Allard N. F., Kielkopf J. F., Chayer P., Dupuis J., Kruk J., Hubeny I., 2003, A\&A, 405, 1153

Herman R., Wallis R. F., 1956, ApJ, 123, 353

Holberg J. B., Kruk J. W., Koester D., Barstow M. A., Burleigh M. R., Sahu M. S., 2003, in de Martino D., Silvotti R., Solheim J. E., Kalytis R., eds, NATO ASIB Proc. 105, White Dwarfs, p. 113

Holweger H., Koester D., Allard N. F., 1994, A\&A, 290, L21

Hylleraas E. A., 1931, Z. Phys., 71, 739 
Jaffé G., 1934, Z. Phys., 87, 535

Kepler S. O., Nelan E. P., 1993, AJ, 105, 608

Kepler S. O., Castanheira B. G., Costa A. F. M., Koester D., 2006, MNRAS, 372, 1799

Kepler S. O., Kleinman S. J., Nitta A., Koester D., Castanheira B. G., Giovannini O., Althaus L., 2007, in Napiwotzki R., Burleigh M. R., eds, ASP Conf. Ser. Vol. 372, White Dwarfs. Astron. Soc. Pac., San Francisco, p. 35

Kepler S. O., Kleinman S. J., Pelisoli I., Peçanha V., Diaz M., Koester D., Castanheira B. G., Nitta A., 2010, AIP Conf. Proc., 1273, 19

Kielkopf J. F., Allard N. F., Decrette A., 2002, Eur. Phys. J. D, 18, 51

Kleinman S. J., 2010, AIP Conf. Proc., 1273, 156

Kleinman S. J. et al., 2004, ApJ, 607, 426

Koester D., 1991, in Vauclair G., Sion E., eds, NATO ASIC Proc. 336, White Dwarfs, p. 343

Koester D., Weidemann V., Zeidler-K. T. E. M., Vauclair G., 1985, A\&A, 142, L5

Koester D., Finley D. S., Allard N. F., Kruk J. W., Kimble R. A., 1996, ApJ, 463, L93

Koester D., Sperhake U., Allard N. F., Finley D. S., Jordan S., 1998, A\&A, 336, 276

Koester D., Kepler S. O., Kleinman S. J., Nitta A., 2009, J. Phys. Conf. Ser., 172, 012006

Koester D. et al., 2009b, A\&A, 505, 441

Koester D., 2010, Mem. Soc. Astron. Ital., 81, 921

Kupka F., Piskunov N., Ryabchikova T. A., Stempels H. C., Weiss W. W., 1999, A\&AS, 138, 119

Kupka F. G., Ryabchikova T. A., Piskunov N. E., Stempels H. C., Weiss W. W., 2000, Balt. Astron., 9, 590

Kurucz R., Bell B., 1995, Atomic Line Data, Technical Report. Smithsonian Astrophysical Observatory, Cambridge, MA
Lemke M., 1997, A\&A, 122, 285

Liebert J., Bergeron P., Holberg J. B., 2005, VizieR Online Data Catalog, 215,60047

Madsen M. M., Peek J. M., 1971, Atomic Data, 2, 171

Margenau H., Lewis M., 1959, Rev. Mod. Phys., 31, 569

Nelan E. P., Wegner G., 1985, ApJ, 289, L31

Piskunov N. E., Kupka F., Ryabchikova T. A., Weiss W. W., Jeffery C. S., 1995, A\&AS, 112, 525

Ramaker D. E., Peek J. M., 1972, J. Phys. B, 5, 2175

Ramaker D. E., Peek J. M., 1973, Atomic Data, 5, 167

Rohrmann R. D., Althaus L. G., Kepler S. O., 2011, MNRAS, 411, 781

Ryabchikova T. A., Piskunov N. E., Kupka F., Weiss W. W., 1997, Balt. Astron., 6, 244

Teller E., 1930, Z. Phys., 61, 458

Tremblay P.-E., Bergeron P., 2009, ApJ, 696, 1755

Tremblay P.-E., Ludwig H.-G., Steffen M., Bergeron P., Freytag B., 2011, A\&AS, 531, L19

Vennes S., Chayer P., Dupuis J., Lanz T., 2005, in Koester D., Moehler S., eds, ASP Conf. Ser. Vol. 334, 14th European Workshop on White Dwarfs. Astron. Soc. Pac., San Francisco, p. 185

Vidal C. R., Cooper J., Smith E. W., 1973, ApJ, 25, 37

Wolff B., Kruk J. W., Koester D., Allard N. F., Ferlet R., Vidal-Madjar A., 2001, A\&A, 373, 674

Yamamoto N., Kato T., Rosmej F. B., 2004, J. Plasma Fusion Res. Ser., 6, 748

Zygelman B., Dalgarno A., 1990, ApJ, 365, 239

This paper has been typeset from a $\mathrm{T}_{\mathrm{E}} \mathrm{X} / \mathrm{L} \mathrm{T} \mathrm{E}_{\mathrm{E}} \mathrm{X}$ file prepared by the author. 